\title{
On a Sharp Transition from Area Law to Perimeter Law in a System of Random Surfaces ${ }^{\star}$
}

\author{
M. Aizenman ${ }^{1, * *}$, J. T. Chayes ${ }^{2, * * *}$, L. Chayes ${ }^{2, * * *}$, J. Fröhlich ${ }^{3}$, and L. Russo ${ }^{4}$ \\ 1 Departments of Mathematics and Physics, Rutgers University, New Brunswick, NJ 08903, USA \\ 2 Department of Physics, Princeton University, Princeton, NJ 08544, USA \\ 3 Theoretische Physik, ETH-Hönggerberg, CH-8093 Zürich, Switzerland \\ 4 Department of Mathematics, Princeton University, Princeton, NJ 08544, USA. Permanent address : \\ Istituto di Matematica dell'Universitá di Modena, I-41100 Modena, Italy
}

\begin{abstract}
We introduce and study a phase transition which is associated with the spontaneous formation of infinite surface sheets in a Bernoulli system of random plaquettes. The transition is manifested by a change in the asymptotic behavior of the probability of the formation of a surface, spanning a prescribed loop. As such, this transition offers a generalization of the bond percolation phenomenon. At low plaquette densities, the probability for large loops is shown to decay exponentially with the loops' area, whereas for high densities the decay is by a perimeter law. Furthermore, we show that the two phases of the three dimensional plaquette system are in a precise correspondence with the two phases of the dual system of random bonds. Thus, if a natural conjecture about the phase structure of the bond percolation model is true, then there is a sharp transition in the asymptotic behavior of the surface events. Our analysis incorporates block variables, in terms of which a non-critical system is transformed into one which is close to a trivial, high or low density, fixed point. Stochastic geometric effects like those discussed here play an important role in lattice gauge theories.
\end{abstract}

\section{Table of Contents}

1. Introduction. Random Surface Analog of Percolation Transition . . . . . . . . . . . . 20

2. Random Plaquette Model in Three Dimensions . . . . . . . . . . . . . . . . . . . 23

i) The Model and Its Dual . . . . . . . . . . . . . . . . . . . . . . . . . . . 23

ii) A priori Bounds . . . . . . . . . . . . . . . . . . . . . . . . . . . 25

iii) Definition of the Critical Points . . . . . . . . . . . . . . . . . . . . . . . . 28

3. Proof of Perimeter Law in the Surface-Dominated Regime . . . . . . . . . . . . . . . 29

i) A Finite Cluster Argument . . . . . . . . . . . . . . . . . . . . . . . . . . 30

ii) A Constructive Proof . . . . . . . . . . . . . . . . . . . . . . . . . . . . 32

iii) A Sharp Constant for Perimeter Law . . . . . . . . . . . . . . . . . . . . . . . 39

* Work supported in part by NSF grants PHY-8301493 (M.A.), PHY-8117463 (J.T.C.) and PHY-8116101 A01 (L.C.)

** Sloan Foundation Research Fellow

*** Address beginning September 1983: Department of Physics, Harvard University, Cambridge, MA 02138, USA 
4. Proof of Area Law in the Dual Percolation Regime . . . . . . . . . . . . . . . . . 42

i) Reduction to a Dense System of Block-Bond Variables . . . . . . . . . . . . . . . 43

ii) A High Density Argument . . . . . . . . . . . . . . . . . . . . . . . . . . 52

iii) A Rescaling Argument . . . . . . . . . . . . . . . . . . 55

5. Summary of Results and Open Problems in Three Dimensions . . . . . . . . . . . . . 59

6. Higher Dimensional Generalizations . . . . . . . . . . . . . . . . . . . . . . . . 61

i) Tree Approximation Bounds for Plaquettes on $\mathbb{Z}^{d}$. . . . . . . . . . . . . . . . . . 61

ii) Cells of Higher Dimension … . . . . . . . . . . . . . . . . . . . . . . . 63

iii) Comments on the Phase Structure in Percolation Models _ . . . . . . . . . . . . 66

7. Relation to Interacting (Gauge) Systems _. . . . . . . . . . . . . . . . . . 66

i) $\left\langle W_{p}\right\rangle$ and the Wilson Loop . . . . . . . . . . . . . . . . . . . . . . . . . 66

ii) A Roughening Transition . . . . . . . . . . . . . . . . . . . . . . 67

\section{Introduction. Random Surface Analog of Percolation Transition}

In this work we present a stochastic-geometric phenomenon which is of interest for reasons stemming from two different origins: gauge theories, and percolation models. The basic system which is studied here is an array of lattice plaquettes which are occupied at random. Even without any interaction, this system exhibits a phase transition, whose global manifestation is in the spontaneous formation of infinite surface sheets.

There has recently been considerable interest in mathematical theories of randomsurfaces. Such theories appear to play an important role in quantum field theory and statistical physics:

(1) (Lattice) gauge theories which are among the central theoretical concepts in elementary particle physics can be reformulated as theories of random surfaces. This can be seen, for example, by performing a high temperature expansion.

(2) Three-dimensional spin systems, like the Ising- or the classical $X Y$ model, and the three-dimensional, classical Coulomb plasma are dual to lattice gauge theories, i.e. theories of random surfaces. Quantities like the specific heat have very natural expressions as averages over random surfaces. An understanding of various theories of random surfaces might therefore provide a clue towards calculating some critical exponents of three-dimensional statistical systems.

(3) Dual resonance models, more precisely string theories are related to theories of random surfaces (at imaginary time, the history of a string is given by a surface belonging to some probability space). String theories might be important as approximate descriptions of gauge theories at large distances.

(4) The physics of crystalline surfaces and of interfaces separating different physical phases leads to the study of models of random surfaces, like the discrete Gaussian, the solid-on-solid model, or a model of self-avoiding random surfaces.

So far, not much is known rigorously about the possible transitions and the critical properties, in particular the existence of continuum (=scaling) limits, of theories of random (lattice) surfaces. The discrete Gaussian and the solid-on-solid model, above the roughening transition, appear to have a trivial continuum limit, a massless field (but a complete proof of this very plausible conjecture is still missing).

Polyakov has recently discussed a continuum theory of random surfaces which appears to be an improved theory of relativistic strings and which poses fascinating mathematical questions $[1,2]$. It represents a promising attempt 
towards constructing an analog of Brownian motion for random surfaces. However its mathematical status and its relevance for the solution of physical problems remain doubtful.

It is therefore of interest to introduce further theories of random surfaces and to analyze the transitions and the critical properties of such theories.

The system which we analyze here is one of random plaquettes - which are the elementary two dimensional cells, with vertices on the $d$ dimensional lattice $\mathbb{Z}^{d}$. (Random arrays of cells of higher dimension are briefly discussed in Sect. 6.) The distribution of plaquette configurations is generated by associating with each plaquette an independent choice of being occupied, or not, with the (homogeneous) probabilities $(p, 1-p)$. That ensemble is, of course, an immediate generalization of the Bernoulli bond percolation model - in which the random elements are lattice edges, which are unit line segments joining nearest neighbor sites of $\mathbb{Z}^{d}$.

It is known that, for $p$ large enough, typical bond configurations of the latter system exhibit infinite connected clusters of occupied bonds, a phenomenon known as percolation. The first question we wish to address is the choice of an interesting analog of the percolation transition for the system of random plaquettes. There is, of course, the phenomenon of formation of infinite clusters of connected plaquettes. However, the two dimensionality of the plaquettes is practically insignificant for this effect. Instead, we propose to study the asymptotic behavior of the probabilities of the events $W_{\gamma}$ defined below.

For each closed loop $\gamma$, consisting of lattice edges, let $W_{\gamma}$ be the set of plaquette configurations for which there exists a collection of occupied plaquettes forming a two dimensional surface whose boundary is exactly $\gamma$. [The relevant topological notions will be made precise in Sect. 2i).] We denote by $\left\langle W_{\gamma}\right\rangle_{p}$ the probability of the set of configurations $W_{\gamma}$ in the ensemble of independent plaquettes which are occupied with the homogeneous probability $p$.

In the next section we shall derive the following pair of "a priori" bounds. For all $0<p<1$ :

$$
\exp \left[-c_{d}(p) \operatorname{Area}(\gamma)\right] \leqq\left\langle W_{\gamma}\right\rangle_{p} \leqq \exp \left[-c_{d}^{\prime}(p) \operatorname{Per}(\gamma)\right]
$$

where $0<c_{d}^{\prime}, c_{d}<\infty$ are explicit, $\gamma$-independent, constants; Area $(\gamma)$ is the minimal area of a surface composed of lattice plaquettes which spans $\gamma$, and $\operatorname{Per}(\gamma)$ is the length (perimeter) of $\gamma$.

We observe that for large loops of simple shape, say rectangular, the two bounds of (1.1) (at a fixed $p$ ) exhibit qualitatively different behavior.

Moreover, for $d \geqq 3$, we prove that the exact behavior of $\left\langle W_{\gamma}\right\rangle_{p}$ does follow an "area law" [like the lower bound in (1.1)] for $p<1 /[2(d-1)]$ and, conversely, a "perimeter law" for $p>$ (const) $d^{-1 / 5}$ [Sect. 6i)].

\section{i) Main Results}

Our main results concern the three dimensional Bernoulli plaquette model.

In three dimensions, the random plaquette system, with density $p$, has as a dual the random bond model, with bond density $(1-p)$. The latter is well known to undergo a percolation transition (as $p$ is varied) - which is manifested in the formation of infinite connected clusters. Actually, one could also consider other notions of critically, for example one which is based on the divergence of the 
expected value of the cluster size. This leads to various critical densities: $p_{c}, \pi_{c}, \varrho_{c}$, and $\hat{\varrho}_{c}$ which are defined in Sect. 2iii). However, it is most natural to expect that all these critical points coincide.

The main results of this paper can be summarized as follows:

Theorem 1.1. For rectangular $(N \times M)$ loops $\gamma$, in a lattice plane, the quantity $\left\langle W_{\gamma}\right\rangle_{p}$ has the following asymptotic behavior:

$$
\left\langle W_{\gamma}\right\rangle_{p} \sim\left\{\begin{array}{lll}
\exp [-\alpha(p) \operatorname{Area}(\gamma)] & \text { for } & p>1-\pi_{c} \\
\exp [-c(p) \operatorname{Per}(\gamma)] & \text { for } & p<1-\hat{\varrho}_{c}
\end{array}\right.
$$

with some $0<\alpha(p), c(p)<\infty$.

The symbol $\left\langle W_{\gamma}\right\rangle \sim e^{-V(\gamma)}$ means here that $\lim _{M, N \rightarrow \infty}-\log \left\langle W_{\gamma}\right\rangle / V(\gamma)=1$, i.e. the constants $\alpha(p)$ and $c(p)$ are actually well defined.

Thus, our results associate the two phases of the radom plaquette system with the opposite phases (in a sense which will be explained below) of the dual bond percolation model. Furthermore, if a natural conjecture on the phase structure of the bond percolation model is correct, then there is a transition from an area law to a perimeter law, which occurs at a single critical value of $p$.

A very interesting question, which is not resolved here, is whether the "surface tension" $\alpha(p)$ vanishes at the critical point. An affirmative answer to this question would be an important step towards the construction of a scaling (continuum) limit.

Theorem 1.1 is proven by supplementing the a priori bounds (1.1) (Proposition 2.3), with the corresponding opposite bounds, provided by Theorem 3.2 and Theorem 4.1. The existence of sharp values for $\alpha$ and $c$ [of which $c(p)$ is the more delicate case] is derived in Proposition 2.4 and Theorem 3.9. In all these results more general loops are also considered.

\section{ii) Further Comments}

We wish to point out that the transition in the behavior of $\left\langle W_{\gamma}\right\rangle_{p}$ has a many-fold relation to the bond percolation transition. One is the duality relation in three dimensions. However, it is also natural to view the transition in (1.2) as a generalization of the transition exhibited by bond percolation.

To cast the bond percolation transition in a more general form, consider the probability that sites $x$ and $y$ are connected by a path of occupied bonds (in a system of independent bonds), which we denote by $\tau_{x, y}$. It is expected (however rigorously proven only for two dimensions [3-5]) that for large $|x-y|, \tau_{x, y}$ behaves as follows:

$$
\tau_{x, y} \sim\left\{\begin{array}{lll}
e^{-m(p)|x-y|}, & m \geqq 0, & \text { for } p<p_{c} \\
c(p)>0 & \text { for } p>p_{c},
\end{array}\right.
$$

where $p_{c}$ is the critical density for the formation of infinite clusters.

Obviously, the event that " $x$ and $y$ are connected" can also be expressed by the statement that among the occupied bonds there is a collection which forms a (finite) line whose boundary is the pair of sites $\{x, y\}$. As such, this event is a clear 
antecedent of $W_{\gamma}$, in a system of lower dimensional geometric objects. There $\{x, y\}$ corresponds to $\gamma$, and the transition exhibited in (1.2) is from an analog of the "area" (here $|x-y|$ ) law to a "perimeter" (here a constant) law.

This analogy provides yet another similarity between the transition discussed here and two dimensional percolation. The dual of the latter is also a bond system. It is known that, in two dimensions, the dual systems are always in opposite phases (except at $p_{c}$ ) [4-7]. Hence the above quoted results indicate an extension of this feature to three dimensions.

Of course, our interest in these pure stochastic-geometric effects is also motivated by discussions of "quark confinement" in gauge models. There, the quantity for which area, versus perimeter, law is of interest is the expected value of "Wilson loop" variables. It turns out that, at least for the abelian $\mathbb{Z}(2)$ gauge model, such a transition can be traced exactly [8] to a geometric effect of the type discussed here, albeit in a system of interacting plaquettes. These, and other, relations with gauge models are described in Sect. 7.

The methods of this paper are based upon two sets of ideas. One is the application of non-perturbative bounds. The other starts with the introduction of block variables, carefully chosen so as to retain the relevant information. Under rescaling, these variables exhibit a simple asymptotic behavior, effectively driving the system away from the critical regime to a trivial fixed point.

A crucial point for the construction of block (bond) variables, which is related to a long-standing conjecture in percolation theory, is the uniqueness of the infinite cluster. Some results on uniqueness are obtained in Sect. 4i) and a brief discussion of this point is found in Sect. 5.

The organization of this paper, and other results which have not been discussed in the introduction, are indicated in the table of contents.

\section{Random Plaquette Model in Three Dimensions}

\section{i) The Model and Its Dual}

We consider the three dimensional lattice $\mathbb{L}=\mathbb{Z}^{3}$. Its elementary $k$ dimensional cells, $k=0,1,2,3$, are the sites (for $k=0$ ), the closed unit intervals, squares, and cubes whose vertices belong to $\mathbb{L}$. We refer to these as the edges $(e)$, plaquettes $(Q)$ and cubes of $\mathbb{L}$.

Let $\mathbb{F}$ be the collection of the plaquettes of $\mathbb{L}$. The space of subsets of $\mathbb{F}$ is $\Omega=\{0,1\}^{\mathbb{F}}$. We regard points of $\Omega$ as configurations of "occupied" plaquettes. Each such configuration is represented by a function $\underline{n}: \mathbb{F} \rightarrow\{0,1\}$, where $n_{Q}=1$ iff $Q$ is occupied.

As mentioned in the introduction, our aim is to discuss surfaces formed by random collections of plaquettes. However, before turning to the stochastic effects, the relevant topological notions should be clarified.

We denote by $\partial Q$ the union of the four edges (of $\mathbb{L}$ ) which form the boundary of the plaquette $Q$. A collection of distinct plaquettes $Q_{1}, \ldots, Q_{k}$ forms a surface, which may either be expressed as the union:

$$
S\left(Q_{1}, \ldots, Q_{k}\right)=\bigcup_{i} Q_{i}
$$




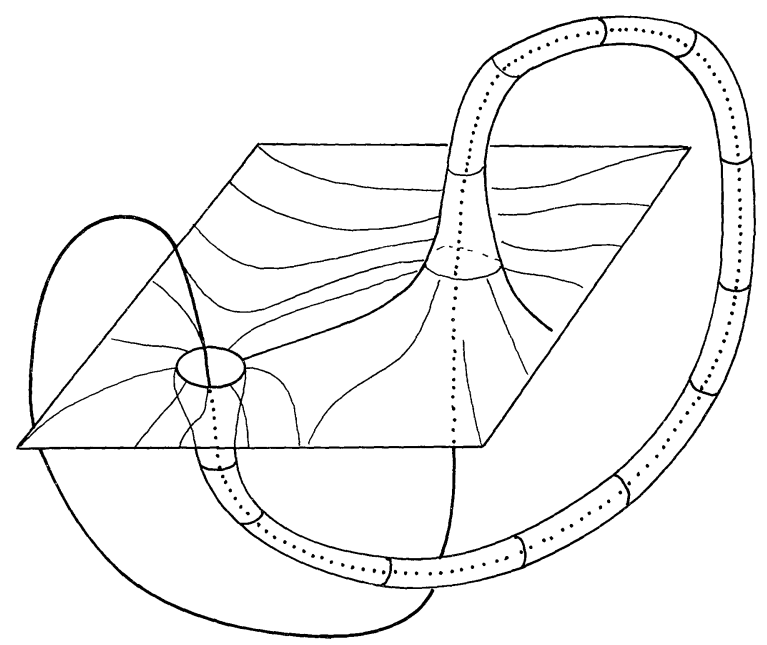

Fig. 1. A plaquette configuration for which there is no orientable surface spanning $\gamma$

or as the closure of their symmetric difference:

$$
S=\overline{Q_{1} \triangle Q_{2} \triangle \ldots \triangle Q_{k}},
$$

where $A \triangle B=A \cup B \backslash A \cap B$. The closure in (2.1) is necessary to avoid spurious subtraction of coinciding edges. The boundary of such a surface is formed by those edges of $\mathbb{L}$ which belong to an odd number of plaquettes in $Q_{1}, \ldots, Q_{k}$. Equivalently

$$
\partial S=\overline{\partial Q_{1} \triangle \partial Q_{2} \triangle \ldots \triangle \partial Q_{k}} .
$$

For any surface $S, \partial S$ is a finite collection of edges. It is easy to see that these edges form a collection of self-avoiding loops. (Throughout this paper, all loops will be understood to be self-avoiding.)

For a given loop $\gamma$, we use the following symbol to denote the collection of plaquette configurations which contain a subset forming an (unbroken) surface which spans $\gamma$ :

$$
W_{\gamma}=\left\{\underline{n} \in \Omega \mid \exists k<\infty \text { and } Q_{1}, \ldots, Q_{k} \text { with } n_{Q_{1}}, \ldots, n_{Q_{k}}=1, \partial S\left(Q_{1}, \ldots, Q_{k}\right)=\gamma\right\} .
$$

Furthermore, we denote by $W_{\gamma}^{0}$ the collection of those configurations for which there is an orientable surface which is spanned by occupied plaquettes, and by $W_{\gamma}^{S}$ those for which one may find a surface which is homeomorphic to a disk.

Clearly,

$$
W_{\gamma} \supset W_{\gamma}^{0} \supset W_{\gamma}^{S}
$$

The less obvious fact that $W_{\gamma} \neq W_{\gamma}^{0}$ is demonstrated by Fig. 1 . We shall now present an alternative characterization of the above events.

With the lattice $\mathbb{L}$ one may associate a dual lattice $\mathbb{L}^{*}=\mathbb{Z}^{3}+\left(\frac{1}{2}, \frac{1}{2}, \frac{1}{2}\right)$, whose sites are the centers of the cubes of $\mathbb{L}$. Each plaquette of $\mathbb{L}$ belongs to the boundary of two cubes. Its dual geometric object is the edge of $\mathbb{L}^{*}$ which joins the centers of these cubes. In order to distinguish edges of the direct lattice $\mathbb{L}$ from those of the dual, $\mathbb{L}^{*}$, henceforth we shall always refer to the former as edges and to the latter 
as bonds $(b)$. In general, to each of the $k$ dimensional elementary cells of $\mathbb{L}$ there corresponds a $3-k$ dimensional cell of $\mathbb{L}^{*}$, which is traversal to it, and intersects it at a single point.

A configuration of plaquettes of $\mathbb{L}$ may alternatively be given by specifying the set of bonds of $\mathbb{L}^{*}$ which correspond to the unoccupied plaquettes. However, it is most convenient to regard the points of $\Omega$ as complementary configurations of plaquettes of $\mathbb{L}$, and bonds of $\mathbb{L}^{*}$ - where a dual bond is occupied whenever the corresponding plaquette is not.

It is very useful to express the events $W_{\gamma}$, defined above, in terms of the occupied bonds (of $\mathbb{L}^{*}$ ). Following is the complete characterization.

Proposition 2.1 [8]. Let $\gamma$ be a loop formed by edges of $\mathbb{L}$, and $S$ a surface (formed by plaquettes) such that $\partial S=\gamma$. Then

$$
\begin{gathered}
W_{\gamma}=\{\underline{n} \in \Omega \mid \text { any closed, or infinite, path along occupied (dual) } \\
\text { bonds of } \underline{n} \text { crosses } S \text { an even number of times }\} .
\end{gathered}
$$

Remarks. 1) This characterization of the event $W_{\gamma}$ depends on the fact that all the closed loops of $\mathbb{L}$ are spanned by the elementary $\partial Q$ 's. That is: for every closed loop on $\mathbb{L}$ there is a finite collection of plaquettes $Q_{1}, \ldots, Q_{k}$ such that $\partial S\left(Q_{1}, \ldots, Q_{k}\right)$ $=\gamma$. (Which really means that, in a discretized sense, $\mathbb{L}$ is simply connected.) Both this statement, and (2.5) are false for a finite lattice with periodic boundary conditions.

2) We shall not provide here the full derivation of Proposition 2.1 - of which we shall use only the easier half. For the completeness of our exposition let us, however, point out that the necessity of the condition in (2.5) is directly implied by the fact that the parity of the number of times a closed path on $\mathbb{L}$ crosses a surface is the same for all the surfaces which $\operatorname{span} \gamma$. This is equivalent to the assertion that any surface with no boundary is crossed an even number of times.

The analog of (2.5) for $W_{\gamma}^{0}$ (which refers to orientable surfaces) is:

Proposition 2.2 [8]. Let $\gamma$ be a loop formed by edges of $\mathbb{L}$, and $S$ an orientable surface (formed by plaquettes) such that $\partial S=\gamma$. Then

$$
\begin{aligned}
& W_{\gamma}^{0}=\{\underline{n} \in \Omega \mid \text { the total flux through } S \text { of any closed, or infinite, } \\
& \text { path along occupied (dual) bonds is zero\}, }
\end{aligned}
$$

where the flux is measured as $(+1)$ for a crossing from the negative to the positive side of $S$, with respect to some orientation, and as $(-1)$ in the opposite case.

The reader is invited to contrast the criteria (2.5) and (2.6) in the example provided by Fig. 1.

For $W_{\gamma}^{S}$ the relevant condition involves chains which are formed by interlocking loops of occupied bonds of $\mathbb{L}^{*}$ (see Fig. 2).

We shall later argue that the transition from an "area law" to a "perimeter law" occurs at the same point for $W_{\gamma}$ and $W_{\gamma}^{0}$, but not $W_{\gamma}^{S}$.

\section{ii) A priori Bounds}

We shall now consider random configurations of plaquettes of $\mathbb{L}$, which are occupied independently of each other with a homogeneous probability $p$. Such an 


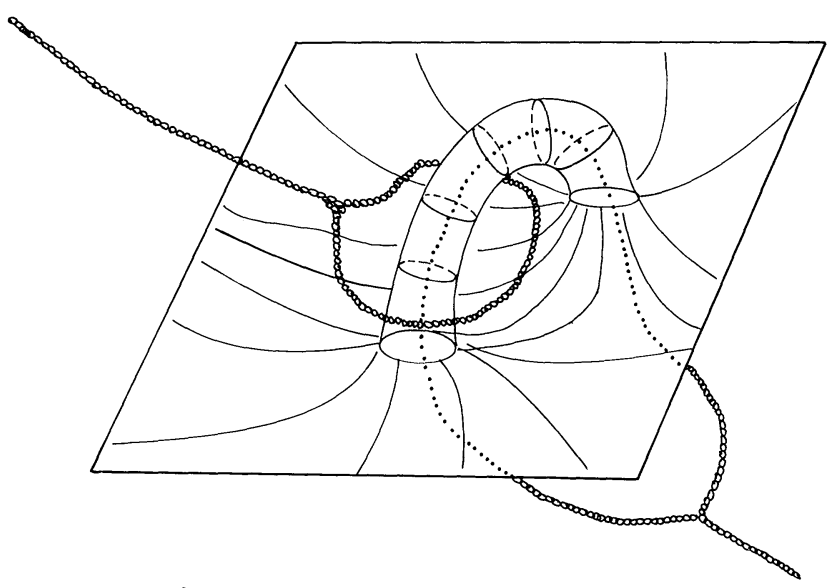

Fig. 2. A configuration in $W_{\gamma}^{0}$ for which there is no disk spanning $\gamma$

ensemble is described by the Bernoulli probability measure $\mu_{p}=\prod_{Q \in F} v_{p}$ on $\Omega$, which is a product of the $(p, 1-p)$ single-plaquette measures, $v_{p}$. Thus $\left\{n_{Q}\right\}$ are independent random variables with the expectation values $\left\langle n_{Q}\right\rangle_{p}=p$. We denote by $\langle-\rangle_{p}=\mu_{p}(-)$ averages (or, for sets, probabilities) with respect to the measure $\mu_{p}$. The quantities $\left\langle W_{\gamma}\right\rangle_{p}$ obey the following very simple bounds.

Proposition 2.3. For every loop $\gamma$, which consists of edges of $\mathbb{L}$ :

$$
\exp [-|\log p| \operatorname{Area}(\gamma)] \leqq\left\langle W_{\gamma}\right\rangle_{p} \leqq \exp [-c(p) \operatorname{Per}(\gamma) / 7],
$$

where Area $(\gamma)=\inf \left\{k \mid \exists Q_{1}, \ldots, Q_{k}\right.$ such that $\left.\partial S\left(Q_{1}, \ldots, Q_{k}\right)=\gamma\right\}$ is the minimal area of a surface spanned by $\gamma, \operatorname{Per}(\gamma)$ is the length (perimeter) of $\gamma$ and $c(p)=\mid \log$ $\left[1-(1-p)^{4}\right] \mid$.

Furthermore, for rectangular loops the upper bound may be replaced by

$$
\left\langle W_{\gamma}\right\rangle_{p} \leqq \exp \{-c(p)[\operatorname{Per}(\gamma)-4]\} .
$$

With suitable modifications of the notion of Area $(\gamma),(2.7)$ (and (2.8)) hold also for $W_{\gamma}^{0}$ and $W_{\gamma}^{S}$.

Proof. 1) Let $S$ be a minimal-area surface which spans $\gamma$ (among the orientable surfaces for $W_{\gamma}^{0}$, and topologically trivial for $W_{\gamma}^{S}$ ). The event $W_{\gamma}$ certainly occurs if all the plaquettes of $S$ are occupied. The probability of this is $p^{\text {Area }(\gamma)}$, which is the lower bound in (2.7).

2) If the event $W_{\gamma}$ occurs, then for each edge $e \in \gamma$ at least one of the four plaquettes to whose boundary the edge $e$ belongs (i.e., in the coboundary of $e$ ) is occupied. We denote such an edge event by $E_{e}$. Its probability is $\mu_{p}\left(E_{e}\right)=1$ $-(1-p)^{4} \equiv \exp [-c(p)]$. Clearly

$$
\left\langle W_{\gamma}\right\rangle_{p} \leqq \mu_{p}\left(\prod_{e \in \gamma} E_{e}\right) \leqq \prod_{k=1}^{N} \mu_{p}\left(E_{e_{k}}\right),
$$


where $e_{1}, \ldots, e_{N}$ is any collection of edges of $\gamma$ with disjoint coboundaries. For rectangular loops this is obtained by deleting one edge at each corner - hence the bound (2.8). For a general (self-avoiding) loop we observe that each edge of $\gamma$ shares a plaquette with at most six other edges (of $\gamma$ ). Thus for (2.9) one may choose at least $N=\operatorname{Per}(\gamma) / 7$. This implies the upper bound in (2.7).

Remark. It is especially instructive to consider the event $R_{e}=\Omega \backslash E_{e}$ - which is the occurence of an elementary ringlet of occupied dual bonds surrounding an edge $e$. The upper bound in (2.7) is a consequence of the fact that if $W_{\gamma}$ occurs then none of the events $R_{e}, e \in \gamma$, occur. One may improve that bound by considering larger encircling loops (on $\mathbb{L})$.

Another result which can be derived using only a priori considerations is the existence, for a large class of loops, of a sharp limiting coefficient for area law. (Obviously, this coefficient vanishes in the perimeter law regime.) In statistical mechanics, the analog of such a constant is known as the surface tension, while in field theories, where the lattice $\mathbb{Z}^{d}$ represents space-time in $(d-1)+1$ dimensions, it is called the string tension. A more detailed discussion of the physical significance of a limiting coefficient for area law can be found in Sect. 7.

The argument makes use of the FKG inequality for the simple case of noninteracting measures. This form, which was first proved by Harris [6], is given below.

Definition. 1) We say that $\underline{n} \leqq \underline{n}^{\prime}$ whenever $n_{Q} \leqq n_{Q}^{\prime}$ for all $Q$. This relation defines a partial order in $\Omega$.

2) An event $A$ is said to be positive if whenever $\underline{n} \in A$ then also $\underline{n^{\prime}} \in A$ for all $\underline{n}^{\prime} \geqq \underline{n}$. An event is said to be negative if its complement is positive.

Lemma 2.4 (FKG inequality) $[6,9]$. If $A$ and $B$ are both positive or both negative events, then

$$
\mu_{p}(A \cap B) \geqq \mu_{p}(A) \mu_{p}(B) .
$$

Definition. Let $\hat{\gamma}$ denote the set of plaquettes which form the minimal surface spanning a planar loop $\gamma$. If $\gamma$ and $\gamma^{\prime}$ are planar loops, then a tiling of $\gamma^{\prime}$ by $\gamma$ is defined to be a partition of $\hat{\gamma}^{\prime}$, into disjoint sets, all but one of which are translates of $\hat{\gamma}$. The distinguished set is called the remainder of the tiling.

Remark. Clearly, for each $\gamma$ and $\gamma^{\prime}$, there exists a (non-unique) maximal tiling of $\gamma^{\prime}$ by $\gamma$, which contains the maximum number, $M\left(\gamma, \gamma^{\prime}\right)$, of translates of $\hat{\gamma}$ as "tiles" of $\hat{\gamma}^{\prime}$. The (unique) number of plaquettes in the remainder of the maximal tiling will be denoted by $r\left(\gamma, \gamma^{\prime}\right)$.

Proposition 2.4. Let $\left(\gamma_{k}\right)$ denote a sequence of planar loops. If for each $k$ the maximal tiling of $\gamma_{N}$ by $\gamma_{k}$ satisfies

$$
\lim _{N \rightarrow \infty}\left[r\left(\gamma_{k}, \gamma_{N}\right) / \operatorname{Area}\left(\gamma_{N}\right)\right]=0,
$$

then

$$
\lim _{k \rightarrow \infty}\left[\log \left\langle W_{\gamma_{k}}\right\rangle_{p} / \operatorname{Area}\left(\gamma_{k}\right)\right]
$$

exists. 
Remark. We note that condition (2.11) is always satisfied for a sequence of rectangular loops, both of whose dimensions tend to infinity. For all such sequences, the limit (2.12) has the same value, which we denote by $-\alpha$.

Proof. Let $n, m \in N$. Choose a maximal tiling of the loop $\gamma_{m}$ by $\gamma_{n}$. If each of the $M\left(\gamma_{n}, \gamma_{m}\right)$ translates of the event $W_{\gamma_{n}}$ occurs and each plaquette in the remainder $r\left(\gamma_{n}, \gamma_{m}\right)$ is occupied, then the event $W_{\gamma_{m}}$ surely occurs. Noting that the former events are all positive and using the FKG inequality, we find

$$
\left\langle W_{\gamma_{m}}\right\rangle_{p} \geqq\left\langle W_{\gamma_{n}}\right\rangle_{p}^{M\left(\gamma_{n}, \gamma_{m}\right)} p^{r\left(\gamma_{n}, \gamma_{m}\right)} .
$$

Then, since $\operatorname{Area}\left(\gamma_{m}\right)=M\left(\gamma_{n}, \gamma_{m}\right) \operatorname{Area}\left(\gamma_{n}\right)+r\left(\gamma_{n}, \gamma_{m}\right)$,

$$
\frac{\log \left\langle W_{\gamma_{m}}\right\rangle_{p}}{\operatorname{Area}\left(\gamma_{m}\right)} \geqq \frac{\log \left\langle W_{\gamma_{n}}\right\rangle_{p}}{\operatorname{Area}\left(\gamma_{n}\right)}+\frac{r\left(\gamma_{n}, \gamma_{m}\right)}{\operatorname{Area}\left(\gamma_{m}\right)}\left[\log p-\frac{\log \left\langle W_{\gamma_{n}}\right\rangle_{p}}{\operatorname{Area}\left(\gamma_{n}\right)}\right] .
$$

Hence for each $n$

$$
\liminf _{m \rightarrow \infty}\left[\frac{\log \left\langle W_{\gamma_{m}}\right\rangle_{p}}{\operatorname{Area}\left(\gamma_{m}\right)}\right] \geqq \frac{\log \left\langle W_{\gamma_{n}}\right\rangle_{p}}{\operatorname{Area}\left(\gamma_{n}\right)} .
$$

Taking $\limsup _{n \rightarrow \infty}$, we obtain the desired result.

The above argument implies also the following bound:

Proposition 2.5. For any rectangular loop $\gamma$

$$
\left\langle W_{\gamma}\right\rangle_{p} \leqq \exp [-\alpha(p) \operatorname{Area}(\gamma)] .
$$

Proof. Clearly, any rectangular loop $\gamma$ may be regarded as an element of a sequence of rectangular loops tending to infinity in the sense of Eq. (2.11). However, by Proposition 2.4, for any such sequence the limit of the left hand side of Eq. (2.15) exists and is (by definition) equal to $-\alpha(p)$.

\section{iii) Definition of the Critical Points}

As explained in Sect. 2i), we regard each $\underline{n} \in \Omega$ as a configuration of mutually exclusive plaquettes of $\mathbb{L}$ and bonds of $\mathbb{L}^{*}$. We shall now define some critical values of $p$, for the measures $\mu_{p}$, which reflect transitions in the global properties of the random bonds. Note that $p$ is the plaquette density. The corresponding bond density is $1-p$.

Each set of occupied bonds decomposes into connected clusters. For a given $\underline{n} \in \Omega$, we denote by $C(i)$ the cluster of occupied bonds which connects to the site $i \in \mathbb{L}^{*} .|C(i)|$ is the number of elements of $C(i)$.

The expected size of the connected cluster of $i \in \mathbb{L}^{*}$ will be denoted by $\langle|C(i)|\rangle_{p}$. By translation invariance of $\mu_{p}$, for every $i \in \mathbb{L}^{*},\langle|C(i)|\rangle_{p}=\langle|C(0)|\rangle_{p}$, where 0 is the origin of $\mathbb{L}^{*}$. We denote by $P_{\infty}(p)$ the $\mu_{p}$-probability that the connected cluster of the origin is infinite, i.e. $P_{\infty}(p)=\mu_{p}(|C(0)|=\infty)$.

Two standard critical points, $\pi_{c}$ and $p_{c}$, are defined by

$$
1-\pi_{c}=\sup \left\{p \in(0,1) \mid\langle|C(0)|\rangle_{p}=\infty\right\},
$$

and

$$
1-p_{c}=\sup \left\{p \in(0,1) \mid P_{\infty}(p)>0\right\} .
$$


Notice that $\pi_{c}$ and $p_{c}$ are critical bond densities.

For the purposes of Sect. 4, we introduce the notion of percolation in layers $\left(\mathbb{L}^{k}\right)$ and quadrant layers $\left(\hat{\mathbb{L}}^{k}\right)$. These are defined as follows :

$$
\mathbb{L}^{k}=\{(x, y, z) \in \mathbb{L} \mid 0 \leqq z \leqq k\}
$$

and

$$
\hat{\mathbb{L}}^{k}=\left\{(x, y, z) \in \mathbb{L}^{k} \mid x, y \geqq 0\right\} .
$$

Let $\mathbb{L}^{* k}$ and $\hat{\mathbb{L}}^{* k}$ be the subsets of $\mathbb{L}^{*}$ which lie entirely "within" $\mathbb{L}^{k}$ and $\hat{\mathbb{L}}^{k}$, respectively.

The clusters $C^{k}(i)$ and $\hat{C}^{k}(i)$ are defined as $C(i)$ above, by restricting consideration only to those bonds which lie entirely within $\mathbb{L}^{* k}$ and $\hat{\mathbb{L}}^{* k}$, respectively. Although $\mathbb{L}^{* k}$ is not translation invariant (in the $z$ direction), for all sites $i \in \mathbb{L}^{* k}$, the quantities $\mu_{p}\left(\left|C^{k}(i)\right|=\infty\right)$ vanish simultaneously as functions of $p$. Hence we define $p_{c}^{k}$ by replacing $P_{\infty}(p)$ in Eq. (2.17) with $P_{\infty}^{k}(p)=\mu_{p}\left(\left|C^{k}(0)\right|=\infty\right)$. Similarly, the quantities $\hat{p}_{c}^{k}$ are defined in terms of $\hat{P}_{\infty}^{k}(p)=\mu_{p}\left(\left|\hat{C}^{k}(0)\right|=\infty\right)$.

Clearly $p_{c}^{k}$ and $\hat{p}_{c}^{k}$ form monotone decreasing sequences. We further define

$$
\varrho_{c}=\lim _{k \rightarrow \infty} p_{c}^{k}
$$

and

$$
\hat{\varrho}_{c}=\lim _{k \rightarrow \infty} \hat{p}_{c}^{k} .
$$

Remark. Simply stated, $\varrho_{c}$ is characterized by the condition that for each $p<1-\varrho_{c}$, the occupied bonds percolate within layers of sufficiently large, yet finite, width. The point $\hat{\varrho}_{c}$ is characterized by a similar condition involving quadrant layers.

Clearly

$$
\pi_{c} \leqq p_{c} \leqq \varrho_{c} \leqq \hat{\varrho}_{c} .
$$

We expect that all these critical points coincide.

Our principal results for three dimensions are that $\left\langle W_{\gamma}\right\rangle_{p}$ obeys a perimeter law for $p>1-\pi_{c}$ and an area law for $p<1-\hat{\varrho}_{c}$.

\section{Proof of Perimeter Law in the Surface-Dominated Regime}

In this section, we prove that $\left\langle W_{\gamma}\right\rangle_{p}$ obeys a perimeter law, for planar loops, whenever $p>1-\pi_{c}$.

Let us first offer an intuitive picture of this behavior. For any $p>1-\pi_{c}$, the expected size of the occupied bond clusters is finite. This suggests that in typical configurations there are unbroken surfaces of occupied plaquettes which extend to infinity in all directions. When this is the case, the event $W_{\gamma}$ occurs if the loop $\gamma$ is connected to such a "pre-existing" surface. Fluctuations along the perimeter suffice to achieve this connection; hence a perimeter law.

We prove the perimeter law by supplementing the a priori upper bound (Proposition 2.3) with a similar lower bound, for which we offer two distinct proofs. 


\section{i) A Finite Cluster Argument}

The dual lattice $\mathbb{L}^{*}=\mathbb{Z}^{3}+\left(\frac{1}{2}, \frac{1}{2}, \frac{1}{2}\right)$ can be expressed as the cartesian product of the " $z$ axis", $K=\left\{\left(\frac{1}{2}, \frac{1}{2}\right)\right\} \times\left(\mathbb{Z}+\frac{1}{2}\right)$, with the " $x y$ plane" $G=\left(\mathbb{Z}^{2}+\left(\frac{1}{2}, \frac{1}{2}\right)\right) \times\left\{\frac{1}{2}\right\}$. We denote by $K^{+}$the (strictly) positive $z$ axis of $\mathbb{L}^{*}$.

Lemma 3.1. Let $D$ denote the event that there is no path of occupied bonds connecting $\mathrm{K}^{+}$with $\mathrm{G}$. Then

$$
\mu_{p}(D)>0
$$

whenever $p>1-\pi_{c}$.

Proof. Let $K_{h}$ denote the part of the $z$ axis $K$ which lies a distance greater than $h$ above the $x y$ plane $G$. Denote by $F_{h}$ the event that there is a path of occupied bonds connecting $K_{h}$ with $G$ (hence $D=\Omega \backslash F_{0}$ ). We shall first show that for each $p>1-\pi_{c}$ there is some finite $h$ such that $\mu_{p}\left(F_{h}\right)<1$.

Let $\tau_{i j}(p)$ denote the probability that $i, j \in \mathbb{L}^{*}$ are connected by a path of occupied bonds. We claim that

$$
\mu_{p}\left(F_{h}\right) \leqq \sum_{\substack{k \in K_{h} \\ j \in G}} \tau_{k j}
$$

In order to see this relation, one should consider the characteristic functions $\chi_{k j}$, which are 1 whenever the sites $k$ and $j$ are connected. Clearly if the event $F_{h}$ occurs, then $\sum \chi_{k j} \geqq 1$. Since $\left\langle\chi_{k j}\right\rangle=\tau_{k j}$, the preceding observation directly implies the bound (3.2).

Similar considerations show that

$$
\begin{aligned}
\langle|C(0)|\rangle & =\sum_{i \in \mathbb{L}^{*}} \tau_{0_{i}} \\
& =\sum_{\substack{k \in K \\
j \in G}} \tau_{k j},
\end{aligned}
$$

the second equality following from translation invariance. Since for $p>1-\pi_{c}$, $\langle|C(0)|\rangle$ is finite, (3.3) implies that

$$
\lim _{h \rightarrow \infty} \sum_{\substack{k \in K_{h} \\ j \in G}} \tau_{k j}=0 .
$$

Combining (3.4) with (3.2), we see that

$$
\mu_{p}\left(\Omega \backslash F_{h}\right)=1-\mu_{p}\left(F_{h}\right)>0
$$

for $h$ large enough.

Next we argue that if (3.5) is valid for some $h<\infty$, then it also holds for $h=0$. It suffices to show that the conditional probability of no connection between the finite segment $K_{0} \backslash K_{h}$ and $G$, given that there is no connection between $K_{h}$ and $G$, is strictly positive. This in fact follows from either of two elementary arguments. First, it is clear that the probability of separating $K_{0} \backslash K_{h}$ from $G$ is greater than the probability that none of the bonds connected to $K_{0} \backslash K_{h}$ are occupied, which is simply $p^{4 h+1}$. Since both the latter event and $\Omega \backslash F_{h}$ are positive in the sense of 
FKG, we obtain

$$
\mu_{p}(D) \geqq p^{4 h+1} \mu_{p}\left(\Omega \backslash F_{h}\right)>0 .
$$

Alternatively, the last step can be demonstrated by a finite fluctuation argument, based on the finiteness of the interaction (which here is zero) without invoking the FKG inequality.

Remark. The event $D$ discussed above can be alternatively characterized as the existence of an infinite surface, formed by occupied plaquettes, which separates the positive $z$ axis from the $x y$ plane.

We shall now turn to the consideration of surfaces with a prescribed boundary.

Theorem 3.2. For each $p>1-\pi_{c}$, there exists a constant $0<c^{\prime}(p)<\infty$ such that for any planar loop $\gamma$

$$
\left\langle W_{\gamma}\right\rangle_{p} \geqq \exp \left[-c^{\prime}(p) \operatorname{Per}(\gamma)\right] .
$$

Proof. Let $\gamma$ be a loop of edges in the $x y$ plane. We denote by $Q_{1}, \ldots, Q_{N}$ those plaquettes in the minimal surface, $S_{\gamma}$, spanning $\gamma$ which share an edge with the loop. The bonds dual to these plaquettes are denoted by $b_{1}, \ldots, b_{N}$. Clearly $N \leqq \operatorname{Per}(\gamma)$.

Consider the $N$ vertical lines which begin at the bonds $b_{i}$ and extend upward parallel to the $z$ axis of $\mathbb{L}^{*}$. Let $D_{i}$ be the event that there is no path of occupied bonds emanating from the $i^{\text {th }}$ such line and intersecting the $x y$ plane. By translation invariance

$$
\mu_{p}\left(D_{i}\right)=\mu_{p}(D)
$$

where $D$ is the event discussed in Lemma 3.1.

The event $\bigcap_{i=1}^{N} D_{i}$ clearly prevents any finite closed path of bonds from interlocking $\gamma$ and hence insures that $W_{\gamma}$ occurs (since, almost surely, there are no infinite paths for $p>1-\pi_{c}$ ). By the FKG inequality

$$
\mu_{p}\left[\bigcap_{i=1}^{N} D_{i}\right] \geqq \prod_{i=1}^{N} \mu_{p}\left(D_{i}\right)=\left[\mu_{p}(D)\right]^{N} \geqq\left[\mu_{p}(D)\right]^{\operatorname{Per}(\gamma)} .
$$

Hence, for $c^{\prime}=\left|\log \mu_{p}(D)\right|$,

$$
\left\langle W_{\gamma}\right\rangle_{p} \geqq \exp \left[-c^{\prime}(p) \operatorname{Per}(\gamma)\right] .
$$

To be more explicit, consider the cylinder obtained by upward translation of the loop $\gamma$. If the event $\bigcap_{i=1}^{N} D_{i}$ occurs, then there is no path of occupied bonds which connects $S_{\gamma}$ with the walls of this cylinder. Let $B_{0}$ be the set of dual sites which lie just "below" $S_{\gamma}$. We now divide the sites of $\mathbb{L}^{*}$ within the cylinder into two disjoint sets: $B_{1}$ consists of those sites which are connected (via a path of occupied bonds) to a point in $B_{0}$, and $B_{2}$ includes those which are not. The plaquettes which separate points of $B_{2}$ from points of $B_{0} \cup B_{1}$ form a manifestly 


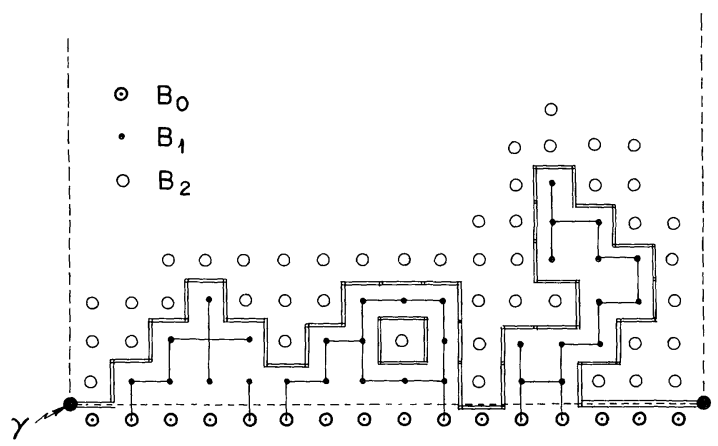

Fig. 3. Cross-section of the surface constructed for Theorem 3.2

orientable surface. One component actually spans $\gamma$; the rest are closed bags (see Fig. 3).

Thus we have shown a stronger result.

Corollary. For $p>1-\pi_{c}$,

$$
\left\langle W_{\gamma}^{0}\right\rangle_{p} \geqq \exp \left[-c^{\prime}(p) \operatorname{Per}(\gamma)\right] .
$$

Theorem 3.2 together with Proposition 2.3 shows that in the high density phase, $p>1-\pi_{c},\left\langle W_{\gamma}\right\rangle_{p}$ has upper and lower perimeter bounds. In Part iii) of this section we prove that the actual behavior is a perimeter law - with a well defined constant $c(p)$. First however we shall describe an alternative proof of Theorem 3.2.

\section{ii) A Constructive Proof}

Here we offer a second proof of perimeter law behavior which, for simplicity, we restrict to the case of rectangular loops. Although this proof is technically more cumbersome than that given in the previous subsection, it has the advantage of actually providing a construction of the surface which may be useful in the study of some of its properties.

Definition. Let $\Delta(a, b, c)$ denote the rectangular solid of dimension $a \times b \times c$

$$
\Delta(a, b, c)=\left\{(x, y, z) \in \mathbb{L}|| x\left|\leqq \frac{a}{2},\right| y \mid \leqq \frac{b}{2}, 0 \leqq z \leqq c\right\} .
$$

Let $S(a, b, c)$ denote the event that there is a surface of occupied plaquettes separating the front $(z=c)$ from the back $(z=0)$ of $\Delta(a, b, c)$ (see Fig. 4).

Note that $S(a, b, c)$ may be (dually) interpreted as the event that there is no bond crossing from the front to the back of $\Delta(a, b, c)$.

We note the following obvious property of the event $S$.

\section{Proposition 3.3.}

$$
\mu_{p}[S(a, b, c)] \geqq \sqrt{\mu_{p}[S(2 a, b, c)]}
$$

and

$$
\mu_{p}[S(a, b, c)] \geqq \sqrt{\mu_{p}[S(a, 2 b, c)]} .
$$




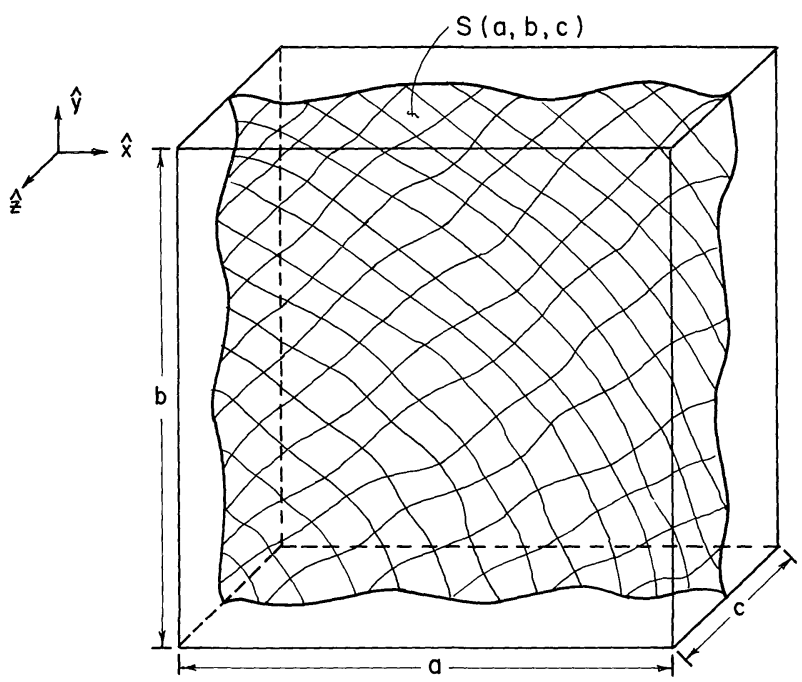

Fig. 4. The event $S(a, b, c)$

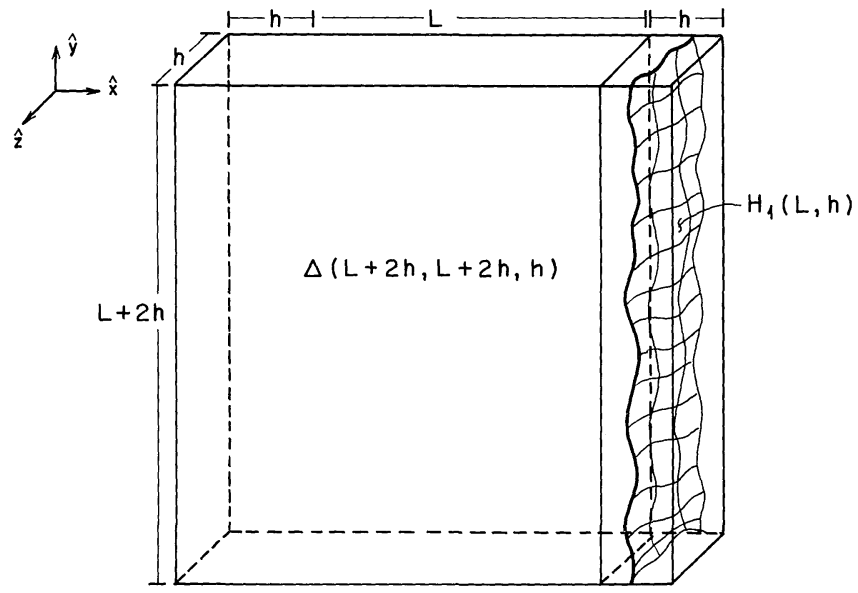

Fig. 5. The box $\Delta(L+2 h, L+2 h, h)$ and the event $H_{1}(L, h)$

Proof. By the $a \leftrightarrow b$ symmetry, the two relations are equivalent. Equation (3.13a) follows from the fact that $S(2 a, b, c)$ is the event that two disjoint translates, $S_{1}$ and $S_{2}$, of $S(a, b, c)$ occur, with the further constraint that two spanning surfaces must join together. Relaxing this constraint is the content of inequality (3.13). We have

$$
\mu_{p}^{2}[S(a, b, c)]=\mu_{p}\left[S_{1}(a, b, c) \cap S_{2}(a, b, c)\right] \geqq \mu_{p}[S(2 a, b, c)] .
$$

We shall now consider events in boxes $A(L+2 h, L+2 h, h)$. Each such box is regarded as consisting of an "inner" part, which is simply $\Delta(L, L, h)$, and an "outer" part, which forms an annulus of width $h$ encasing the $L \times L$ square. The outer annulus consists of four overlapping copies of $\Delta(L+2 h, h, h)$, each of which is referred to as a boundary region (see Fig. 5). 


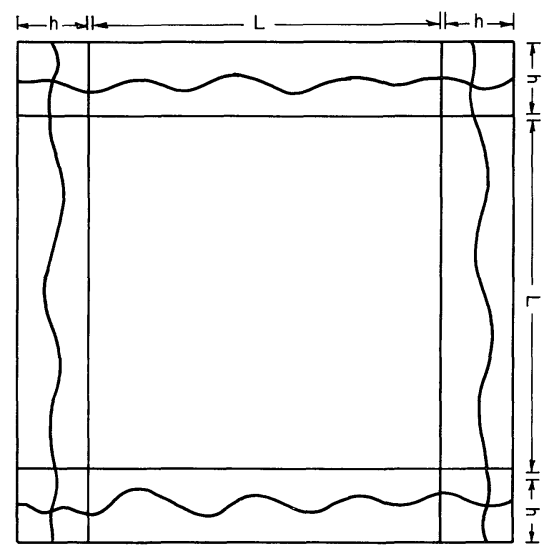

Fig. 6. Two dimensional projection of the event $P(L, h)$

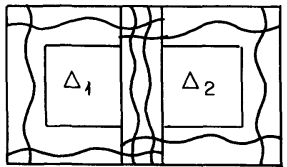

$\mathbf{a}$

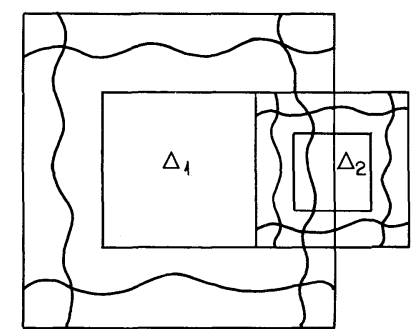

Fig. $7 \mathbf{a}$ and $\mathbf{b}$. Connection of plaquette events

Definition. We denote by $H_{1}(L, h)$ the event that there is a surface of occupied plaquettes separating the $x=L$ face of $\Delta(L+2 h, L+2 h, h)$ from its $x=L+h$ face and lying entirely in the boundary region

$$
\left\{(x, y, z) \in \mathbb{L}|L \leqq x \leqq L+h,| y \mid \leqq \frac{L}{2}+h, 0 \leqq z \leqq h\right\} .
$$

Similarly, let $H_{2}(L, h), \ldots, H_{4}(L, h)$ denote the analogous events in the other three boundary regions. We note that the $H_{i}(L, h)$ are equivalent to the event $S(L+2 h, h, h)$ by translations and rotations.

We call

$$
P(L, h)=S(L+2 h, L+2 h, h) \cap\left[\bigcap_{i=1}^{4} H_{i}(L, h)\right]
$$

the rescaled plaquette event. Pictorially, it is convenient to represent $P(L, h)$ by its two dimensional projection in the $x y$ plane, as in Fig. 6.

Remark. $P(L, h)$ is referred to as a plaquette event because when two boxes of the same dimensions, $\Delta_{1}$ and $\Delta_{2}$, are placed next to each other in such a way that the adjacent boundary regions coincide, the occurrence of the plaquette event in both boxes implies that there is an unbroken surface in the larger box, $\Delta_{1} \cup \Delta_{2}$, separating the front from the back (see Fig. 7a). 


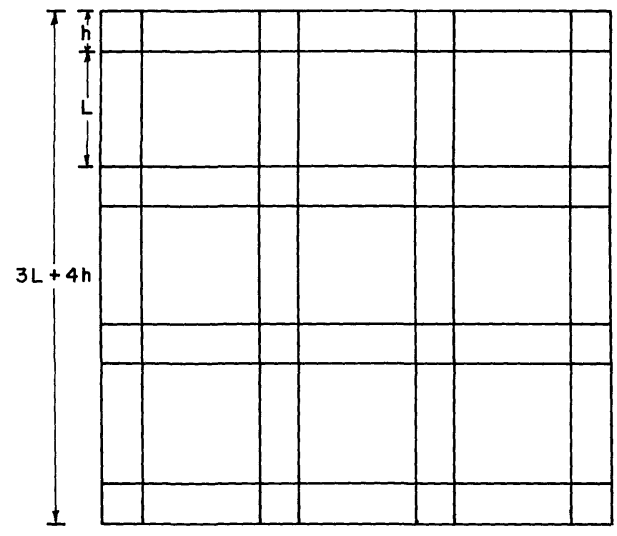

Fig. 8. Nine overlapping translates of $\Delta(L+2 h, L+2 h, h)$

To insure connection of plaquette events of different length scale, with $L_{1}>L_{2}$ and $h_{1}>h_{2}$, we place the plaquette as shown in Fig. 7b, so that the boundary region of the smaller box lies entirely within the adjacent boundary region of the larger box. Thus the boundary region of the larger box $\Delta_{1}$ intersects the interior (i.e., non-boundary) region of the smaller box $\Delta_{2}$.

The crucial property of the plaquette events is their behavior under rescaling. As is demonstrated by the following lemma, if a plaquette event is sufficiently probable, then rescaling enhances its probability.

Lemma 3.4. Let $\lambda<1$ and $\alpha=\frac{1}{2}\left(3^{-4}\right)$. If

$$
\mu_{p}[P(L, h)] \geqq 1-\alpha \lambda
$$

for some $h<(3 / 4) L$, then

$$
\mu_{p}[P(3 L, 2 h)] \geqq 1-\alpha \lambda^{2} .
$$

Proof. First place nine boxes of the form $\Delta(L+2 h, L+2 h, h)$ adjacent to each other with boundary regions overlapping (as in Fig. 8) to obtain a large square of the form $\Delta(3 L+4 h, 3 L+4 h, h)$.

If plaquette events occur in all of the small boxes, then there is a surface spanning the large square, i.e. the event $S(3 L+4 h, 3 L+4 h, h)$ occurs. Hence

$$
\mu_{p}[S(3 L+4 h, 3 L+4 h, h)] \geqq 1-9 \alpha \lambda .
$$

This implies that

$$
\mu_{p}[S(3 L+4 h, 3 L+4 h, 2 h)] \geqq 1-(9 \alpha \lambda)^{2},
$$

since the event $S(3 L+4 h, 3 L+4 h, 2 h)$ surely occurs if $S(3 L+4 h, 3 L+4 h, h)$ takes place in either the front half or the back half of $\Delta(3 L+4 h, 3 L+4 h, 2 h)$.

To achieve the event $P(3 L, 2 h)$, we also need the boundary events $H_{i}(3 L, 2 h)$ which, as remarked previously, are simply translates of the event $S(3 L+4 h, 2 h, 2 h)$. 
Thus by the FKG inequality,

$$
\mu_{p}\left[\bigcap_{i=1}^{4} H_{i}(3 L, 2 h)\right] \geqq \mu_{p}^{4}\left[H_{1}(3 L, 2 h)\right]=\mu_{p}^{4}[S(3 L+4 h, 2 h, 2 h)] .
$$

The term on the right hand side of (3.20) can be bounded below by noting that $3 L$ $+4 h \geqq 8 h$ and applying Proposition 3.3 (twice), to obtain

$$
\mu_{p}^{4}[S(3 L+4 h, 2 h, 2 h)] \geqq \mu_{p}[S(3 L+4 h, 3 L+4 h, 2 h)] .
$$

Using Eqs. (3.19)-(3.21), we obtain the desired lower bound

$$
\begin{aligned}
\mu_{p}[P(3 L, 2 h) 1 & \geqq 1-2(9 \alpha \lambda)^{2} \\
& =1-\alpha \lambda^{2} .
\end{aligned}
$$

Remark. Iterating the rescaling procedure $k$ times, we find that if $\mu_{p}[P(L, h)] \geqq 1$ $-\alpha \lambda$ for $h \leqq(3 / 4) L$, then

$$
\mu_{p}\left[P\left(3^{k} L, 2^{k} h\right)\right] \geqq 1-\alpha \lambda^{2^{k}} .
$$

Hence the probability of the plaquette events approaches one very rapidly. Moreover, the rescaled plaquette events occur in asymptotically flat regions, in the sense that height/length goes to zero with $k$. The relative size of the boundary region also approaches zero with $k$.

For a given loop $\gamma$, we shall use plaquette events of scales up to the size of the loop to construct a spanning surface of occupied plaquettes. In order to do this, we must first show that, if $p>1-\pi_{c}$, there exists a scale $L$ such that the first rescaled plaquette event, $P(3 L, L)$, satisfies $\mu_{p}[P(3 L, L)]>1-\alpha$. This will be shown to follow from a property of the event $S(3 L, 3 L, L)$ which was first derived (in a quite general context) by Kesten [10].

Proposition 3.5. If $p>1-\pi_{c}$, then $\forall \varepsilon>0, \exists L$ such that $\mu_{p}[P(3 L, L)]>1-\varepsilon$.

Proof. Kesten [10] has shown that if $p>1-\pi_{c}$, then $\lim _{L \rightarrow \infty} \mu_{p}[S(3 L, 3 L, L)]=1$.

By reasoning analogous to that in Proposition 3.3 and Lemma 3.4, it is easy to show that

$$
\mu_{p}[P(L, L)] \geqq \mu_{p}^{7 / 3}[S(3 L, 3 L, L)] \rightarrow 1 .
$$

Grouping four translates of the event $P(L, L)$ into a larger block (see Fig. 9) and using the FKG inequality, we obtain the estimate

$$
\mu_{p}[P(3 L, L)] \geqq \mu_{p}^{4}[P(L, L)] .
$$

Hence $\mu_{p}[P(3 L, L)]$ also tends to 1 with $L$.

We now offer a second proof of Theorem 3.2 for the case of rectangular loops. Proof (of Theorem 3.2). Let $M$ and $N$ denote the dimensions of the loop $\gamma$ which is taken to be centered in the $x y$ plane of $\mathbb{L}$. Let $L$ be the smallest length scale for which $\mu_{p}[P(3 L, L)] \geqq 1-\alpha / 2$.

We tile the inner rim of the loop $\gamma$ with translates of the plaquette events $P(3 L, L)$ as shown in Fig. 10 . 


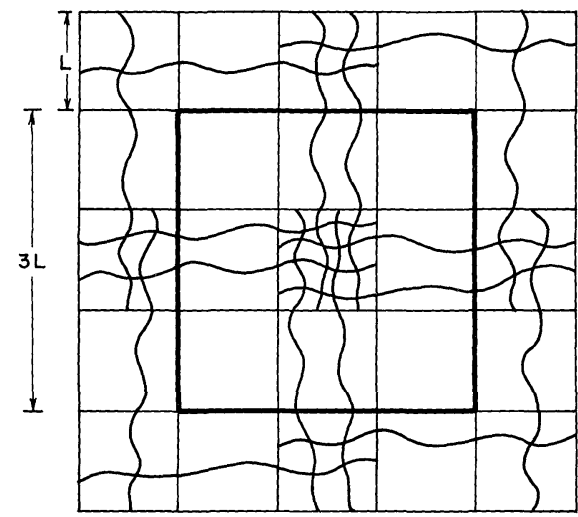

Fig. 9. Four overlapping translates of $P(L, L)$

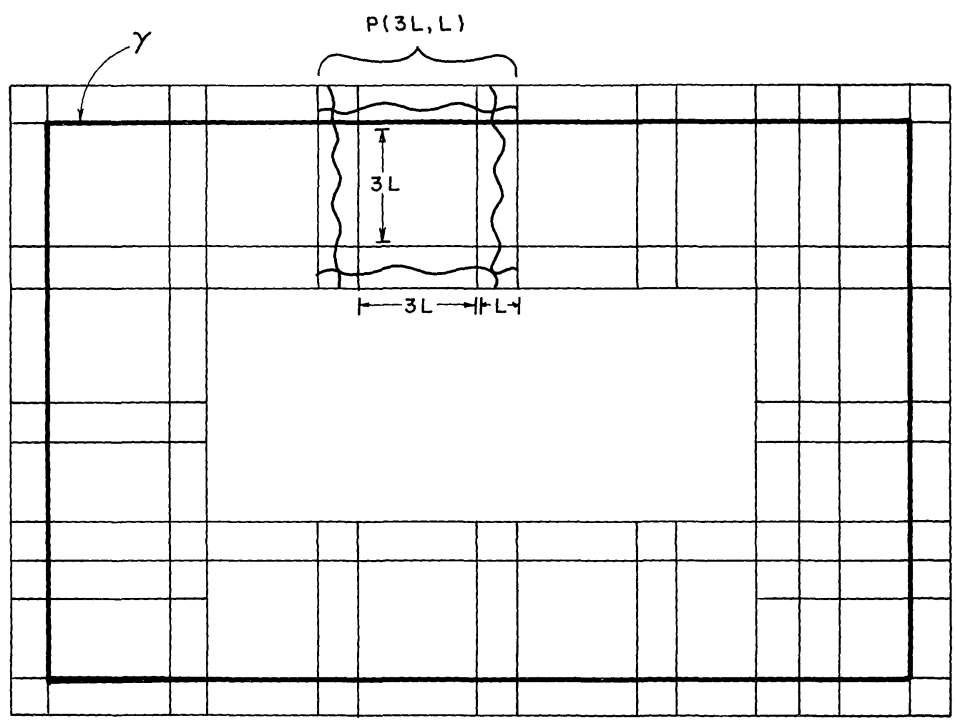

Fig. 10. Tilling of the inner rim of $\gamma$ (only one plaquette event is shown)

In this tiling, we place the "plaquettes" $P(3 L, L)$ so that their outer boundaries (of width $L$ ) extend beyond the loop $\gamma$. Whenever possible, adjacent boundary regions of the plaquettes are taken to coincide. Since $M$ and $N$ are not generally the proper multiples of $L$, the boundary regions of the "tiles" at the corners of $\gamma$ may be required to overlap the interior (non-boundary) regions of adjacent tiles. It is clear that this construction assures the connection of surfaces of adjacent plaquettes.

Let $n_{1}$ denote the number of translates of $P(3 L, L)$ required to tile the inner rim. Clearly $n_{1} \leqq 2(M+N) / 3 L=\operatorname{Per}(\gamma) / 3 L$. [We have discarded the extra length that is obtained from the boundary regions of $P(3 L, L)$ and we have counted the corners twice.] Let us denote the simultaneous occurrence of all $n_{1}$ plaquette events by $\omega_{1}$. 


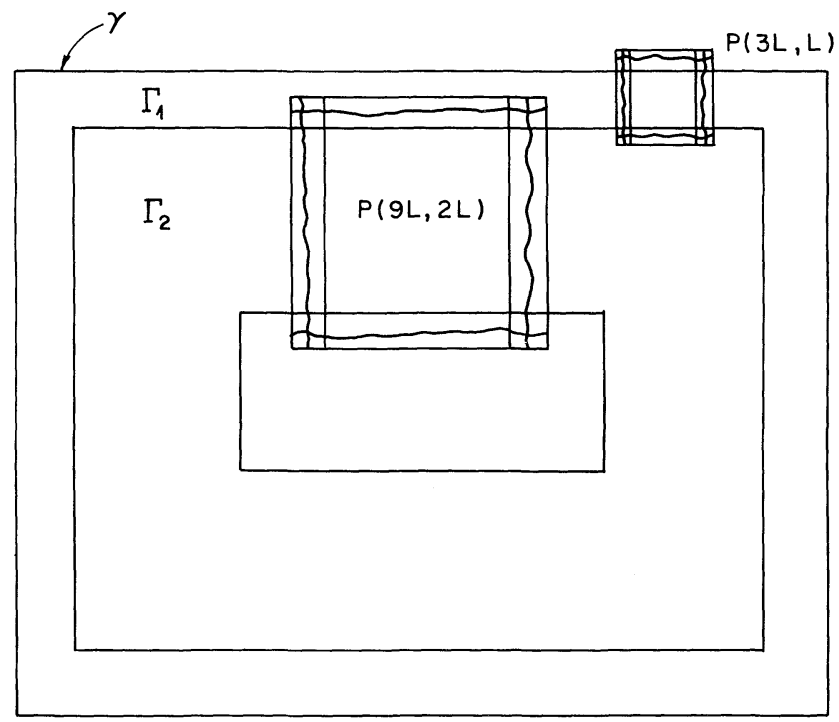

Fig. 11. The annular regions $\Gamma_{1}$ and $\Gamma_{2}$ with representative tiles

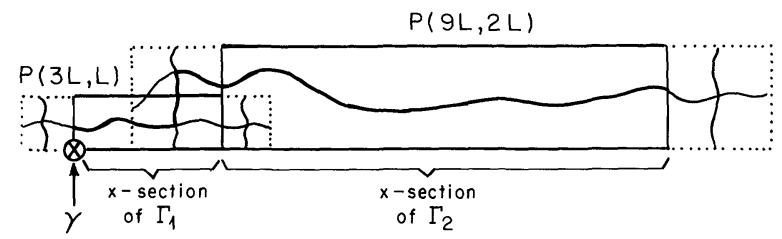

Fig. 12. Cross-section of the surfaces in the annular regions $\Gamma_{1}$ and $\Gamma_{2}$

Using the FKG inequality, we have

$$
\mu_{p}\left(\omega_{1}\right) \geqq\left[1-\frac{1}{2} \alpha\right]^{n_{1}} \geqq\left[1-\frac{1}{2} \alpha\right]^{(1 / 3)(\operatorname{Per}(\gamma) / L)} .
$$

Note that the event $\omega_{1}$ implies that there is an unbroken surface separating the front $(z=L)$ of the annular region

$$
\Gamma_{1}=\left\{(x, y, z) \in \mathbb{L}\left|\frac{M}{2}-3 L \leqq\right| x\left|\leqq \frac{M}{2}, \frac{N}{2}-3 L \leqq\right| y \mid \leqq \frac{N}{2}, 0 \leqq z \leqq L\right\}
$$

(see Fig. 11) from the back $(z=0)$.

Next we tile the second annular region

$\Gamma_{2}=\left\{(x, y, z) \in \mathbb{L}\left|\frac{M}{2}-12 L \leqq\right| x\left|\leqq \frac{M}{2}-3 L, \frac{N}{2}-12 L \leqq\right| y \mid \leqq \frac{N}{2}-3 L, 0 \leqq z \leqq 2 L\right\}$

(see Fig. 11) with the plaquette events $P(9 L, 2 L)$.

This tiling produces a surface in the annulus $\Gamma_{2}$. Moreover, the plaquette events $P(9 L, 2 L)$ interest the annulus $\Gamma_{1}$ in such a way the two surfaces in $\Gamma_{1}$ and $\Gamma_{2}$ are joined to form a larger surface. This is illustrated in Fig. 12. 
The second tiling event, which we shall call $\omega_{2}$, requires the simultaneous occurrence of $n_{2}$ plaquette events $P(9 L, 2 L)$, where $n_{2} \leqq 2(M+N-12 L) / 9 L$ $<\operatorname{Per}(\gamma) / 9 L$.

We continue the procedure, defining tiling events $\omega_{3}, \ldots, \omega_{s}$ for the annular regions $\Gamma_{3}, \ldots, \Gamma_{s}$ until the interior is exhausted. (This happens quite rapidly; the procedure terminates when $s$ satisfies

$$
\left.\sum_{n=1}^{s} 3^{n} \geqq \min \left\{\frac{M}{L}, \frac{N}{L}\right\} .\right)
$$

Each event $\omega_{k}$ is the intersection of $n_{k} \leqq \operatorname{Per}(\gamma) / 3^{k} L$ translates $P\left(3^{k-1}(3 L), 2^{k-1} L\right)$. Hence

$$
\mu_{p}\left(\omega_{k}\right) \geqq\left[1-\alpha \sqrt{\frac{1}{2}} 2^{k}\right]^{\operatorname{Per}(\gamma) / 3^{k} L} .
$$

Finally, define the event $\omega_{0}$ to be the existence of a wall of occupied plaquettes extending outward from the loop $\gamma$ to a height $z=L$. This event has probability $p^{L \operatorname{Per}(\gamma)}$. Clearly the simultaneous occurrence of $\omega_{0}, \ldots, \omega_{s}$ implies $W_{\gamma}$. Thus, again using FKG, we have the estimate

$$
\begin{aligned}
\left\langle W_{\gamma}\right\rangle_{p} & \geqq \mu_{p}\left(\bigcap_{k=0}^{s} \omega_{k}\right) \geqq \prod_{k=0}^{s} \mu_{p}\left(\omega_{k}\right) \\
& \geqq\left[p^{L \operatorname{Per}(\gamma)}\right]\left(\prod_{k=1}^{s}\left[1-\alpha \sqrt{\frac{1}{2}} 2^{k}\right]^{\operatorname{Per}(\gamma) / 3^{k} L}\right) \\
& \geqq\left[p^{L \zeta^{1 / L}}\right]^{\operatorname{Per}(\gamma)} \equiv \exp \left[-c^{\prime}(p) \operatorname{Per}(\gamma)\right] .
\end{aligned}
$$

Here $\zeta>0$ denotes the infinite product

$$
\zeta=\prod_{k=1}^{\infty}\left[1-\alpha \sqrt{\frac{1}{2}} 2^{k}\right]^{\left(1 / 3^{k}\right)},
$$

and hence is independent of the loop $\gamma$.

Remark. The surface constructed by the above procedure is obviously orientable and lies in the upper half plane.

\section{iii) A Sharp Constant for Perimeter Law}

In Proposition 2.4 it was demonstrated that for a large class of planar loops the coefficient of area law tends to a sharp constant.

Such a strong result is not expected if $\left\langle W_{\gamma}\right\rangle_{p}$ obeys a perimeter law period. For example, by serrating a given loop, we may easily double or triple the perimeter. Yet, this should not drastically alter the probability that the loop is spanned.

We can show that for $p>1-\pi_{c}$, the coefficient of length law for loops with "smooth" perimeters (i.e., loops with a fixed number of corners) tends to a sharp constant.

Definition. Let $\Lambda_{N}$ denote the cube $\{(x, y, z) \in \mathbb{L}|| x|| y,|| z \mid, \leqq N\}$.

Let $C_{N}$ denote the event that there exists a surface $S$ of occupied plaquettes in $\Lambda_{N}$, such that $\partial S$ consists of the line segment $|x| \leqq N, y=0, z=0$ and edges which lie 


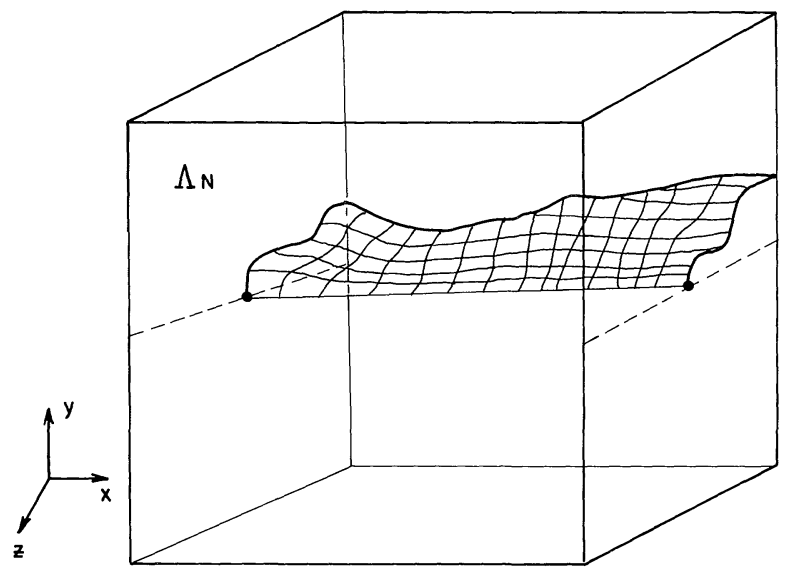

Fig. 13. The event $C_{N}$

exclusively in $\partial \Lambda_{N}$. Dually, this means that no circuit of occupied bonds in $\Lambda_{N}$ encircles the $x$-axis with odd linking number (see Fig. 13).

Proposition 3.6. For every $p \in[0,1]$,

exists.

$$
\lim _{N \rightarrow \infty} \frac{\mu_{p}\left(C_{N}\right)}{N}
$$

Proof. Let $N, M$ be integers. Take $N>M$ and let $\left[\frac{N}{M}\right]$ denote the largest integer smaller than $\frac{N}{M}$. If $C_{N}$ occurs, then $\left[\frac{N}{M}\right]$ translates of the event $C_{M}$ occur. This is easy to see by placing disjoint translates of the smaller cube centered along the line segment $|x| \leqq N, y=0, z=0$. Thus

$$
\mu_{p}\left(C_{N}\right) \leqq \mu_{p}\left(C_{M}\right)^{[N / M]}
$$

or

$$
\frac{\log \mu_{p}\left(C_{N}\right)}{N} \leqq \frac{\log \mu_{p}\left(C_{M}\right)}{M}+O\left(\frac{1}{N}\right) .
$$

Taking $\lim _{N \rightarrow \infty}$ sup and $\lim$ inf, respectively, we obtain the desired result.

Remark. In the surface dominated regime, it is clear that the above limit is finite; it shall be denoted by $-c(p)$.

Next, we consider a "dressed up" version of the cube event.

Let $\bar{C}_{N}$ denote the intersection of $C_{N}$ with three other events : (1) the top face of $\Lambda_{N}$ is separated from the bottom by two surfaces of occupied plaquettes, one lying entirely above, and the other entirely below, the $x y$-plane; (2) and (3) analogous events for the other two pairs of opposing faces. 
Proposition 3.7. For $p>1-\pi_{c}$

$$
\lim _{N \rightarrow \infty} \frac{\log \mu_{p}\left(\bar{C}_{N}\right)}{N}=-c(p) .
$$

Proof. Clearly $\mu_{p}\left(C_{N}\right) \geqq \mu_{p}\left(\bar{C}_{N}\right)$.

However, in order for $\bar{C}_{N}$ to occur, it is sufficient that $C_{N}$ occur along with six renormalized plaquette events, the probabilities of which tend to one. A lower bound is obtained using FKG. In fact, it is not difficult to show that, for $N$ sufficiently large, there exists a constant $K$ such that

$$
\mu_{p}\left(\bar{C}_{N}\right) \geqq \mu_{p}\left(C_{N}\right)\left(1-e^{-K N}\right) .
$$

Definition. Let $T_{N, L}$ denote the tube $\{(x, y, z) \in \mathbb{L}|| x|\leqq L ;| y|| z \mid,=N\}$.

Denote by $t_{N, L}$ the event that the top slab $(z=N)$ of $T_{N, L}$ is separated from the bottom $(z=-N)$ by a surface of occupied plaquettes which is pinned to the line segment $|x| \leqq L, y=0, z=0$.

Proposition 3.8. For $p>1-\pi_{c}$

$$
\lim _{N \rightarrow \infty} \lim _{M \rightarrow \infty} \frac{\log \mu_{p}\left(t_{N, L}\right)}{L}=-c(p) .
$$

Proof. The proof of this proposition is analogous to that of the previous two propositions.

Theorem 3.9. Let $\gamma_{0}$ be any fixed loop and let $\left(\gamma_{M}\right)$ be a sequence of loops which are scaled up copies of $\gamma_{0}$. Then

$$
\lim _{k \rightarrow \infty} \frac{\log \left\langle W_{\gamma_{M}}\right\rangle_{p}}{\operatorname{Per}\left(\gamma_{M}\right)}=-c(p)
$$

whenever $p>1-\pi_{c}$.

Proof. Let $\Delta$ denote the (fixed) number of corners of the loops $\gamma_{M}$. For $M$ sufficiently large, the occcurrence of the event $W_{\gamma_{M}}$ implies that at least $\frac{\operatorname{Per}\left(\gamma_{M}\right)}{N}$ $-2 \Delta$ disjoint translates of event $C_{N}$ occur. Thus, by Proposition $3.6,\left\langle W_{\gamma_{M}}\right\rangle_{p}$ is asymptotically bounded above by $\exp \left[-c(p) \operatorname{Per}\left(\gamma_{M}\right)\right]$.

On the other hand, we may surround the segments of the loop $\gamma_{M}$, excluding corners, with tubes of the form $T_{N, L_{i}}$ (so that $\left.\sum_{i} L_{i}=\operatorname{Per}\left(\gamma_{m}\right)-2 \Delta N\right)$. In any configuration in which the events $t_{N, L_{2}}$ occur in all of the tubes, the event $W_{\gamma_{M}}$ is achieved if (1) all the plaquettes in cubes $\Delta_{N}$ centered at each corner are occupied, and (2) a surface of renormalized plaquettes of initial height $N$ spans the new loop formed by the tubes. Thus, using FKG and noting that the probabilities of the plaquette events approach one exponentially in $N$, we see that asymptotically $\left\langle W_{\gamma_{M}}\right\rangle_{p}$ is also bounded below by $\exp \left[-c(p) \operatorname{Per}\left(\gamma_{M}\right)\right]$. 


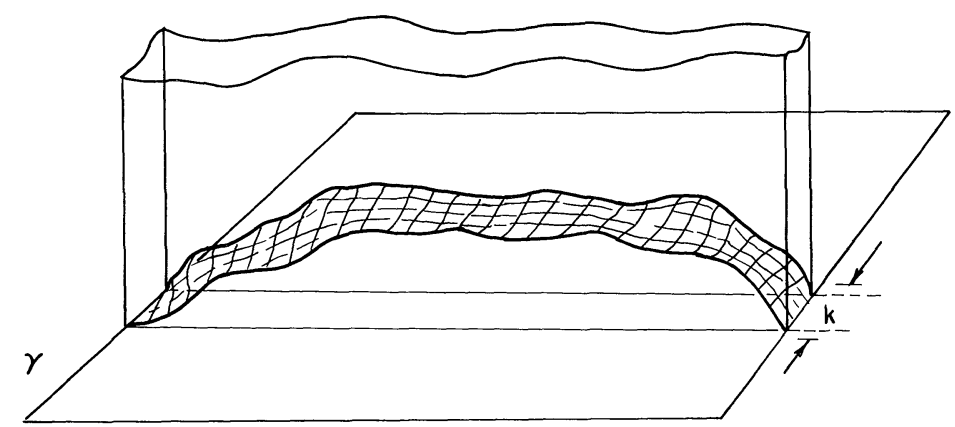

Fig. 14. A bridge of occupied plaquettes

\section{Proof of Area Law in the Dual Percolation Regime}

In this section, we show that for $p<1-\hat{\varrho}_{c},\left\langle W_{\gamma}\right\rangle_{p}$ obeys an area law. This is accomplished by supplementing the a priori lower bound of Proposition 2.3 with an upper bound of the same form. For the purposes of this section, we restrict attention to rectangular loops. (As indicated by Proposition 2.4, only these loops need be considered to obtain a more general result.)

Since our proof of area law is rather involved, let us first discuss the general scheme.

Whenever $p<1-\hat{\varrho}_{c}$, bonds percolate in some quadrant layer of a finite thickness $k$. We show in Sect. 4i) that this enables us to construct, within this layer, "renormalized" bond variables which occur with probability very close to one.

Should the event $W_{\gamma}$ occur, it is clear that inside each $k$-layer which cuts the loop, opposite sides of $\gamma$ are joined by a "bridge" of plaquettes whose projected width is $k$ (see Fig. 14).

To be more explicit, define a pin, $d_{x, y}^{(k)}$, to be a set of points in the direct lattice layer $\mathbb{L}^{k}$ given by

$$
d_{x, y}^{(k)}=\left\{(\bar{x}, \bar{y}, \bar{z}) \in \mathbb{L}^{k} \mid \bar{x}=x, \bar{y}=y\right\} .
$$

As is clear from Fig. 14, the intersection of a rectangular loop $\gamma$ centered in the $x z$ plane with $\mathbb{L}^{k}$ consists of two pins separated by the width of the loop.

A bridge between the pins $d_{x_{1}, 0}^{(k)}$ and $d_{x_{2}, 0}^{(k)}$ is a surface $S$ of plaquettes within the layer $\mathbb{L}^{k}$ such that the intersection of the boundary $\partial S$ with the interior of $\mathbb{L}^{k}$ is simply the pins $d_{x_{1}, 0}^{(k)}$ and $d_{x_{2}, 0}^{(k)}$. In other words, the boundary of the bridge consists of $d_{x_{1}, 0}^{(k)}$ and $d_{x_{2}, 0}^{(k)}$ and some edges of the layer $\mathbb{L}^{k}$ which lie entirely in the planes $z=0$, or $z=k$.

We denote the event of a bridge of occupied plaquettes between $d_{x_{1}, 0}^{(k)}$ and $d_{x_{2}, 0}^{(k)}$ by $\beta^{(k)}\left(x_{1}, x_{2}\right)$. Using suitably generalized two-dimensional techniques, we prove in Sects. 4ii)-iii) the following upper bound which leads to the area law.

Theorem 4.1. For each $p<1-\hat{p}_{c}^{k}$, there exists a constant $m(p)>0$ such that, for any $x_{1}$ and $x_{2}$,

$$
\mu_{p}\left[\beta^{(k)}\left(x_{1}, x_{2}\right)\right] \leqq \exp \left[-m(p)\left|x_{1}-x_{2}\right|\right]
$$


The proof of this result is the subject of the rest of the following sections. Here let us demonstrate that it implies the area law.

Corollary. Suppose $p<1-\hat{\varrho}_{c}$. Then there is a constant $\alpha(p)>0$ such that, for any rectangular loop $\gamma$,

$$
\left\langle W_{\gamma}\right\rangle_{p}<\exp [-\alpha(p) \operatorname{Area}(\gamma)] .
$$

Proof. Let $k$ be the smallest integer for which $p<1-\hat{p}_{c}^{k}$. Let $M$ and $N$ denote the lengths of the sides of $\gamma$. We shall first prove (4.3) for cases in which $M / k$ is an integer.

Consider the layer of width $M$ which contains (and is normal to) the loop $\gamma$. Let us divide this layer into exactly $M / k$ translates of $\mathbb{L}^{k}$. If $W_{\gamma}$ occurs, then within any layer of thickness $k$, a translate of the event $\beta^{(k)}(0, N)$ must occur. Since there are $M / k$ disjoint layers, we have

$$
\left\langle W_{\gamma}\right\rangle_{p} \leqq \exp (-[m(p) N][M / k]) \equiv \exp [-\hat{c}(p) \operatorname{Area}(\gamma)] .
$$

By Proposition 2.4, the sequence of rectangular loops of size $(n k \times N)$ has a well defined surface tension which satisfies $\alpha(p) \geqq \hat{c}(p)>0$. Therefore, by Proposition 2.5, for any rectangular loop $\gamma$, we have the bound

$$
\left\langle W_{\gamma}\right\rangle_{p} \leqq \exp [-\alpha(p) \text { Area }(\gamma)] \text {. }
$$

\section{i) Reduction to a Dense System of Block-Bond Variables}

Most events considered in this section are best characterized in terms of bond configurations. The density of occupied bonds is $q=1-p$. To emphasize the relevant parameter, we denote

$$
\tilde{\mu}_{q}(\cdot)=\mu_{1-q}(\cdot)=\mu_{p}(\cdot) .
$$

We first prove an elementary probabilistic lemma which is used at several points in this section.

Definition. Let $\Gamma=\{0,1\}^{\mathbb{N}}$ be a configuration space with associated Bernoulli measure $m_{p}$. For any configuration $\underline{n} \in \Gamma$, we define the sphere

$$
S^{N}(\underline{n})=\left\{\underline{n}^{\prime} \in \Gamma\left|\sum_{i \in \mathbb{N}}\right| n_{i}-n_{i}^{\prime} \mid \leqq N\right\}
$$

Let $A \subset \Gamma$ be an event. We define

$$
I^{N} A=\left\{\underline{n} \in \Gamma \mid S^{N}(\underline{n}) \subset A\right\},
$$

and

$$
E^{N} A=\left\{\underline{n} \in \Gamma \mid S^{N}(\underline{n}) \cap A \neq \emptyset\right\} .
$$

Thus $I^{N} A$ consists of those configurations for which the event $A$ occurs and is stable to modification of any set of up to $N$ variables $\left\{n_{i}\right\}$. The configurations in $E^{N} A(\subset A)$ include those for which a change of some set of no more than $N$ variables $\left\{n_{i}\right\}$ produces the event $A$. We note that

$$
\Gamma \backslash I^{N} A=E^{N}(\Gamma \backslash A) .
$$


Lemma 4.2. Let $0 \leqq p^{\prime} \leqq p$. For any positive event $A$ :

$$
\begin{gathered}
m_{p}(A) \geqq\left(p-p^{\prime}\right)^{N} m_{p^{\prime}}\left(E^{N} A\right), \\
m_{p}\left(I^{N} A\right) \geqq 1-\frac{\left[1-m_{p^{\prime}}(A)\right]}{\left(p-p^{\prime}\right)^{N}} .
\end{gathered}
$$

Proof. Let $\Delta=p-p^{\prime}$. The key to (4.11) is to realize the measure $m_{p}$ as being generated in two steps. First, one draws a random configuration $\underline{n}^{\prime}\left(n_{i}^{\prime}=0,1\right)$ with density $p^{\prime}=p-\Delta$. In the second step, an independent configuration $\underline{n}^{\prime \prime}$ is drawn with density $\Delta /\left(1-p^{\prime}\right)$. Thus $\left(\underline{n}^{\prime}, \underline{n}^{\prime \prime}\right)$ are distributed with measure $m_{p} \times m_{\Delta /\left(1-p^{\prime}\right)}$. The union of the two configurations, that is

$$
\underline{n}=\max \left(\underline{n}^{\prime}, \underline{n}^{\prime \prime}\right)
$$

has a Bernoulli distribution with density

$$
p^{\prime}+\left(1-p^{\prime}\right) \frac{\Delta}{1-p^{\prime}}=p
$$

This is most easily seen by regarding the event $\left\{n_{i}=1\right\}$ as the disjoint union of the events $\left\{n_{i}^{\prime}=1\right\}$ and $\left\{n_{i}^{\prime}=0, n_{i}^{\prime \prime}=1\right\}$.

Thus $m_{p}(A)$ is the probability that $A$ occurs in the random configuration $\underline{n}$. To bound this probability below, we observe that if the weaker event $E^{N} A$ occurs in $\underline{n}^{\prime}$, then there is a set (which depends on $\underline{n}^{\prime}$ ), of no more than $N$ sites, which if all occupied in $\underline{n}^{\prime \prime}$ would produce the event $A$ in $\underline{n}$. This proves that

$$
m_{p}(A) \geqq\left(\frac{\Delta}{1-p^{\prime}}\right)^{N} m_{p^{\prime}}\left(E^{N} A\right),
$$

which directly implies (4.11).

Relation (4.12) may be proved by a similar argument. This time it is the measure $m_{p}$, which should be viewed as being generated in two steps. First, a random configuration $\underline{n}$ at density $p$ is drawn. Next one draws an independent configuration $\underline{n}^{\prime \prime}$ at density $1-(\Delta / p)$. (For small $\Delta$, this may be regarded as a configuration containing a low density distribution of "holes", which are used to remove sites from the configuration $\underline{n}$.) Thus $\left(\underline{n}, \underline{n}^{\prime \prime}\right)$ are distributed with measure $m_{p} \times m_{1-(\Delta / p)}$. The intersection of the two configurations.

$$
\underline{n}^{\prime}=\min \left(\underline{n}, \underline{n}^{\prime \prime}\right) \text {, }
$$

has a Bernoulli distribution with density

$$
p(1-\Delta / p)=p^{\prime}
$$

This follows from the fact that the event $\left\{n_{i}^{\prime}=1\right\}$ is the intersection of the events $\left\{n_{i}=1\right\}$ and $\left\{n_{i}^{\prime \prime}=1\right\}$.

Thus $m_{p^{\prime}}(\Gamma \backslash A)$ is the probability that $A$ does not occur in the random configuration $\underline{n}^{\prime}$. We note that if $I^{N} A$ does not occur in $\underline{n}$ (which is more probable than $\Gamma \backslash A$ ), then there is a set, depending on $\underline{n}$, of no more than $N$ sites, which if none are occupied in $\underline{n}^{\prime \prime}$, guarantee that the event $A$ will not occur in $\underline{n}^{\prime}$. Thus

which implies (4.12).

$$
m_{p^{\prime}}(\Gamma \backslash A) \geqq\left(\frac{\Delta}{p}\right)^{N} m_{p}\left(\Gamma \backslash I^{N} A\right),
$$




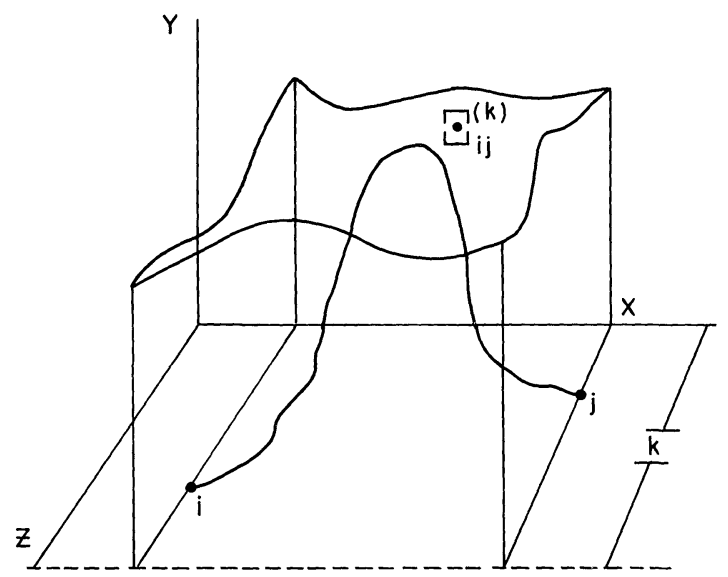

Fig. 15. The event $\Xi_{i j}^{(k)}$

Alternatively (4.12) may be proved from (4.11) by a duality argument. We note that for any negative event $B \subset \Gamma$, Eq. (4.11) becomes

$$
m_{p^{\prime}}(B) \geqq \Delta^{N} m_{p}\left(E^{N} B\right) \text {. }
$$

This follows from (4.11) by regarding the measure $\tilde{m}_{q}=m_{1-p}$ as a Bernoulli measure at density $q$ on distributions of "holes". In this language, the event $B$ is positive and thus (4.11) gives

$$
\tilde{m}_{q+\Delta}(B) \geqq \Delta^{N} \tilde{m}_{q}\left(E^{N} B\right),
$$

which is equivalent to (4.17).

Since $\Gamma \backslash A$ is indeed negative, Eqs. (4.10) and (4.17) imply

$$
1-m_{p^{\prime}}(A) \geqq \Delta^{N} m_{p}\left(E^{N}(\Gamma \backslash A)\right)=\Delta^{N}\left[1-m_{p}\left(I^{N} A\right)\right],
$$

which proves (4.12).

Next we establish some properties of the infinite cluster for $q \geqq \hat{p}_{c}^{k}$.

Definition. Let $i, j \in \mathbb{L}^{* k}$ have $y$ coordinates equal to zero and the $x$ coordinates $i_{1}, j_{1}$, with $i_{1}<j_{1}$. We denote by $\Xi_{i j}^{(k)}$ the event that $i$ and $j$ are connected by a path of occupied bonds contained entirely within the region

$$
Q_{i j}^{(k)}=\left\{(x, y, z) \in \mathbb{L}^{* k} \mid i_{1} \leqq x \leqq j_{1}, y \geqq 0\right\}
$$

(see Fig. 15).

Lemma 4.3. For every $q>\hat{p}_{c}^{k}, \tilde{\mu}_{q}\left(\Xi_{i j}^{(k)}\right)$ is bounded below by a constant $a(q)>0$ uniformly in $|i-j|$.

Proof. Let $q-\hat{p}_{c}^{k} \equiv 2 \Delta>0$. At bond density $q^{\prime}=q-\Delta$, both $i$ and $j$ are connected to infinity in their respective quadrants,

$$
Q_{i,+}^{(k)}=\left\{(x, y, z) \in \mathbb{L}^{* k} \mid x \geqq i_{1}, y \geqq 0\right\},
$$




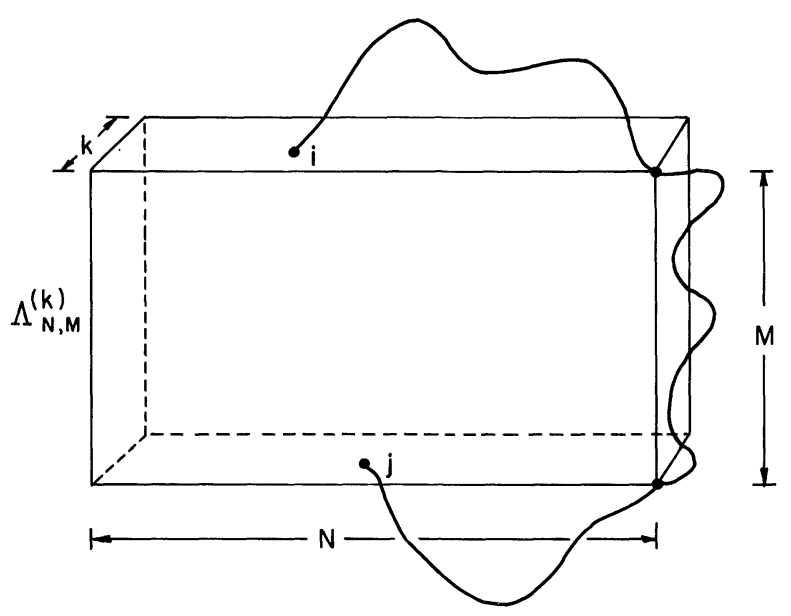

Fig. 16. Connection between $i, j \in \partial \Lambda_{N, M}^{(k)}$ by path in $\mathbb{L}^{* k} \backslash \Lambda_{N, M}^{(k)}$

and

$$
Q_{j,--}^{(k)}=\left\{(x, y, z) \in \mathbb{L}^{* k} \mid x \leqq j_{1}, y \geqq 0\right\},
$$

with probability not smaller than $\left[\hat{P}_{\infty}^{k}\left(p^{\prime}\right)\right]^{2}$ (by FKG), with $p^{\prime}=1-q^{\prime}$. This event implies that there exists a path of occupied bonds in $Q_{i j}^{(k)}=Q_{i,+}^{(k)} \cap Q_{j,-}^{(k)}$ emanating from $i$ which overlaps a similar path emanating from $j$. [Two paths are said to overlap if they have at least one vertex with the same $(x, y)$ coordinate, so that their projections intersect.]

The occurrence of two such overlapping paths clearly implies that the configuration is in $E^{k} \Xi_{i j}^{(k)}$. Hence we have

$$
\tilde{\mu}_{q-\Delta}\left(E^{k} \Xi_{i j}^{(k)}\right) \geqq\left[\hat{P}_{\infty}^{k}\left(p^{\prime}\right)\right]^{2} .
$$

This yields, by Lemma 4.2(a),

$$
\tilde{\mu}\left(\Xi_{i j}^{(k)}\right) \geqq \Delta^{k}\left[\hat{P}_{\infty}^{k}\left(p^{\prime}\right)\right]^{2}>0
$$

Corollary. Let $\Lambda_{N, M}^{(k)}$ be an $N \times M \times k$ rectangular box

$$
\Lambda_{N, M}^{(k)}=\left\{(x, y, z) \in \mathbb{L}^{* k}|| x|\leqq N,| y \mid \leqq M\right\},
$$

and let $\partial \Lambda_{N, M}^{(k)}$ denote its boundary

$$
\partial \Lambda_{N, M}^{(k)}=\left\{(x, y, z) \in \mathbb{L}^{* k}|| x|=N,| y \mid=M\right\} .
$$

If $i, j \in \partial \Lambda_{N, M}^{(k)}$, then with $\tilde{\mu}_{q}$-probability bounded below by $[a(q)]^{3}$, there is a path of occupied bonds in $\mathbb{L}^{* k} \backslash \Lambda_{N, M}^{(k)}$ connecting $i$ with $j$ (see Fig 16).

Theorem 4.4. Suppose $q>\hat{q}_{c}^{k}$. Then the infinite cluster in $\mathbb{L}^{* k}$ is unique.

Proof. Let $i, j \in \mathbb{L}^{* k}$. By countable subadditivity of $\tilde{\mu}_{q}(\cdot)$, it is sufficient to show that the set of configurations for which both $i$ and $j$ are connected to infinity within $\mathbb{L}^{* k}$ and $i \notin C^{k}(j)^{1}$ has measure zero. We denote this set of configurations by $A$.

1 This is a slight abuse of notation. By $i \in C^{k}(j)$, we mean that $i$ is the endpoint of a bond in $C^{k}(j)$ 


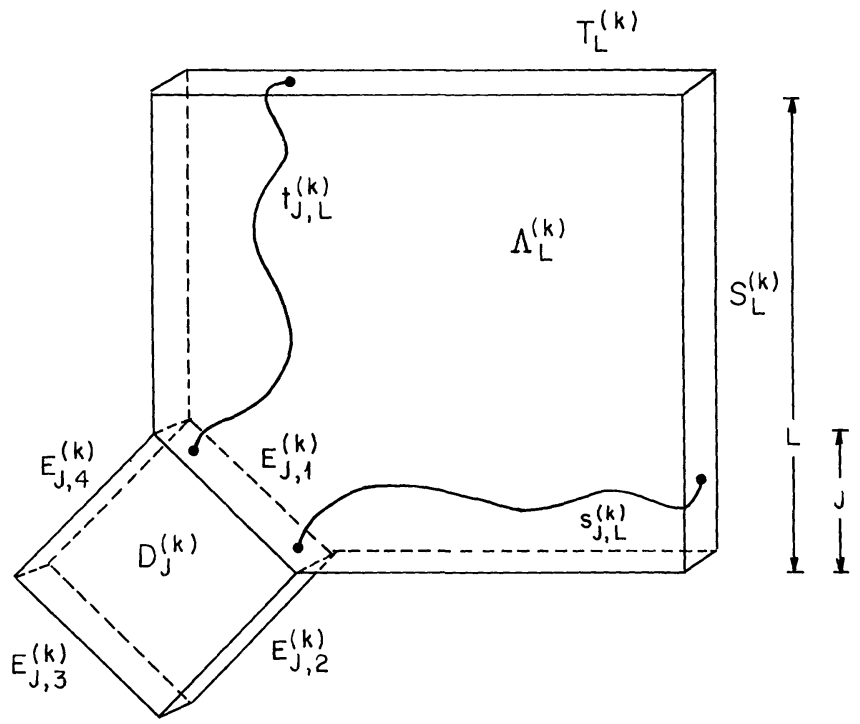

Fig. 17. The events $s_{J, L}^{(k)}$ and $t_{J, L}^{(k)}$

Let $\left(\Delta_{N} \subset \mathbb{L}^{* k}\right), N=1,2, \ldots$, be a nested sequence of rectangular boxes (i.e., $\left.\Delta_{N} \subset \Delta_{N+1}\right)$ such that $i, j \in \Delta_{1}$ and $\lim _{N \rightarrow \infty} \Delta_{N}=\mathbb{L}^{* k}$.

For $\underline{n} \in \Omega$, let $C_{N}(l)$ denote the set of points connected to $l \in \Delta_{N}$ by occupied bonds, all of which lie within $\Delta_{N}$.

Let $A_{N}$ be the event that $i$ and $j$ are both connected to $\partial \Delta_{N}$ but are not connected to each other within $\Delta_{N}$

$$
A_{N}=\left\{\underline{n} \in \Omega \mid C_{N}(i) \cap \partial \Delta_{N} \neq \emptyset, C_{N}(j) \cap \partial \Delta_{N} \neq \emptyset, i \notin C_{N}(j)\right\} .
$$

Note that $A_{N+1} \subset A_{N}$ and $A=\bigcap_{N=1}^{\infty} A_{N}$. Hence by continuity of the measures

$$
\tilde{\mu}_{q}(A)=\lim _{N \rightarrow \infty} \tilde{\mu}_{q}\left(A_{N}\right) .
$$

By the corollary to Lemma 4.3, the conditional probability of $A$ given that $A_{N}$ occurs obeys

$$
\tilde{\mu}_{q}\left(A \mid A_{N}\right) \leqq 1-[a(q)]^{3} .
$$

However, since $A \cap A_{N}=A$, we have $\tilde{\mu}_{q}\left(A \mid A_{N}\right)=\tilde{\mu}_{q}(A) / \tilde{\mu}_{q}\left(A_{N}\right)$.

Thus

$$
\tilde{\mu}_{q}(A) \leqq\left(1-a^{3}\right) \tilde{\mu}_{q}\left(A_{N}\right) .
$$

Taking the limit $N \rightarrow \infty$ and noting that $a^{3}>0$, we obtain the desired result

$$
\mu_{p}(A)=0 \text {. }
$$

Next, we introduce some of the sets and events necessary for the construction of the renormalized bond lattice.

Definition (see Fig. 17). Let $E_{J, 1}^{(k)}$ be the set of sites in $\mathbb{L}^{* k}$ given by

$$
E_{J, 1}^{(k)}=\left\{(x, y, z) \in \mathbb{L}^{* k} \mid x, y \geqq 0, y=J-x\right\} .
$$


We denote by $E_{J, 2}^{(k)}, E_{J, 3}^{(k)}$, and $E_{J, 4}^{(k)}$, the reflections of $E_{J, 1}^{(k)}$ about the planes $y=0$, $x=0$ and $y=-x$, respectively. The union of the above four sets forms the boundary of a "diamond," which we shall denote by $D_{J}^{(k)}$.

Take $L>J$ and let $\Lambda_{L}^{(k)}$ be the $L \times L \times k$ box defined by

$$
\Lambda_{L}^{(k)}=\left\{(x, y, z) \in \mathbb{L}^{* k} \mid 0 \leqq x \leqq L, 0 \leqq y \leqq L\right\} .
$$

Let $S_{L}^{(k)} \subset \partial \Lambda_{L}^{(k)}$ be the right side of $\Lambda_{L}^{(k)}$, i.e.

$$
S_{L}^{(k)}=\left\{(x, y, z) \in \Lambda_{L}^{(k)} \mid x=L\right\} .
$$

Similarly, let $T_{L}^{(k)} \subset \partial \Lambda_{L}^{(k)}$ be the top of $\Lambda_{L}^{(k)}$ :

$$
T_{L}^{(k)}=\left\{(x, y, z) \in \Lambda_{L}^{(k)} \mid y=L\right\} .
$$

We define $s_{J, L}^{(k)}$ to be the event that $E_{J, 1}^{(k)}$ is connected to $S_{L}^{(k)}$ by a path of occupied bonds which lie entirely within $\Lambda_{L}^{(k)}$. Let $t_{J, L}^{(k)}$ denote the event of an analogous connection between $E_{J, 1}^{(k)}$ and $T_{L}^{(k)}$.

Proposition 4.5. For every $q>\hat{p}_{c}^{k}$, there is a J large enough so that $\tilde{\mu}_{q}\left(s_{J, L}^{(k)}\right)=\tilde{\mu}_{q}\left(t_{J, L}^{(k)}\right)$ is arbitrarily close to one (provided that $L>J$ ).

Proof. At bond density $q>\hat{p}_{c}^{k}$, if $J$ is sufficiently large, then $E_{J .1}^{(k)}$ is connected to infinity within the quadrant layer $\hat{\mathbb{L}}^{* k}$ with $\tilde{\mu}_{q}$-probability as close to one as desired. This implies that the probability that $E_{J, 1}^{(k)}$ is disconnected from $S_{L}^{(k)} \cup T_{L}^{(k)}$ tends to zero as $J$ gets large.

The probability that $E_{J, 1}^{(k)}$ is not connected to $S_{L}^{(k)}$ is $1-\tilde{\mu}_{q}\left(s_{J, L}^{(k)}\right)$, which is also the probability that $E_{J, 1}^{(k)}$ is not connected to $T_{L}^{(k)}$. By the FKG inequality, the probability that $E_{J, 1}^{(k)}$ is not connected to either of these two sets is larger than $\left[1-\tilde{\mu}_{q}\left(s_{J, L}^{(k)}\right)\right]^{2}$, and as we have seen, this probability is arbitrarily close to zero for sufficiently large $J$.

Definition. Let $\mathbb{V}^{*}$ be the lattice

$$
\mathbb{V}^{*}=\{n(\hat{x}+\hat{y})+m(\hat{x}-\hat{y}) \mid n, m \in \mathbb{Z}\} \equiv\left\{(n+m, n-m) \in \mathbb{R}^{2} \mid n, m \in \mathbb{Z}\right\},
$$

which is oriented at $45^{\circ}$ with respect to the lattice $\mathbb{L}^{* k}$. For each $i \in \mathbb{V}^{*}$ we denote by $E_{J, 1}^{(k)}(i), D_{J}^{(k)}(i), \Lambda_{L}^{(k)}(i), S_{L}^{(k)}(i)$, etc. the "horizontal" translates of the sets $E_{J, 1}^{(k)}, D_{J}^{(k)}, \Lambda_{L}^{(k)}$, $S_{L}^{(k)}$ etc. by the vector $i L$.

Remark. The set of diamonds $D_{L}^{(k)}(i)$ forms a partition of the lattice $\mathbb{L}^{* k}$. In the centers of these diamonds are the smaller diamonds $D_{J}^{(k)}(i)$. One may regard the latter as the sites of a "coarser" lattice, which is oriented at $45^{\circ}$ relative to $\mathbb{L}^{* k}$ and has the Euclidean spacing $\sqrt{2} L$ (see Fig. 18).

We shall now associate with each bond configuration on $\mathbb{L}^{* k}$ a configuration of "occupied" bonds on $\mathbb{V}^{*}$ - which represent renormalized bond events.

Definition. Let $i, j \in \mathbb{V}^{*}$, with $|i-j|=\sqrt{2}$ be a set of neighboring sites of $\mathbb{V}^{*}$. We regard $b=\{i, j\}$ as a bond of $\mathbb{V}^{*}$, and denote the event

$$
\begin{aligned}
h_{b}^{(k)}=\{ & \left\{\underline{n} \in \Omega \mid D_{J}^{(k)}(i) \text { and } D_{J}^{(k)}(j)\right. \text { are connected by some path of } \\
& \text { occupied bonds of } \mathbb{L}^{* k} \text { which lies entirely within } \\
& \left.D_{L}^{(k)}(i) \cup D_{L}^{(k)}(j)\right\} .
\end{aligned}
$$




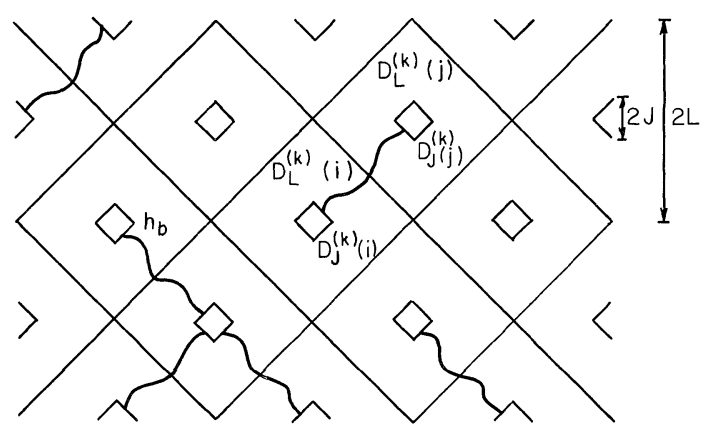

Fig. 18. The partition of the lattice $\mathbb{L}^{* k}$

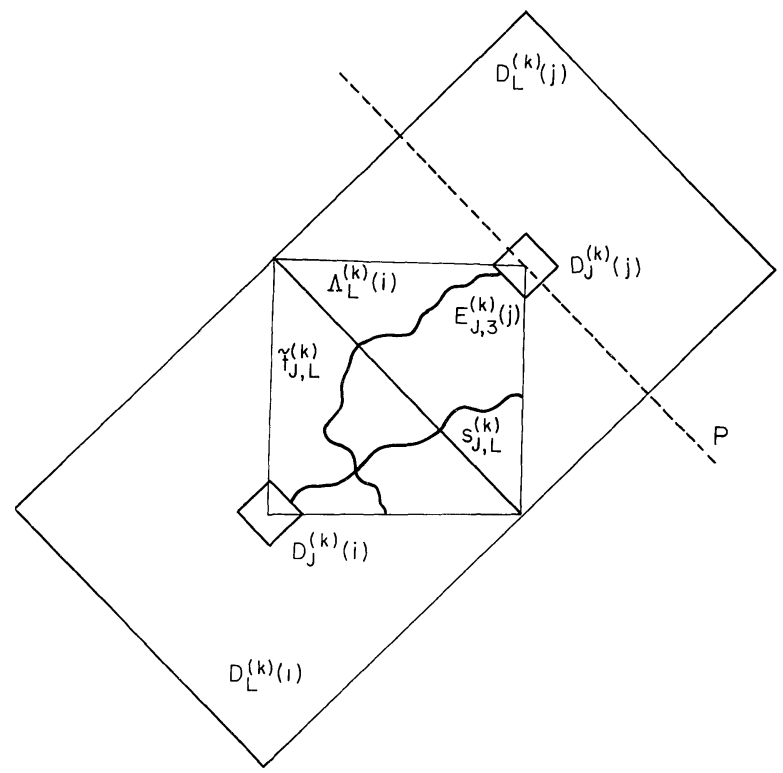

Fig. 19. The events $s_{J, L}^{(k)}$ and $\tilde{t}_{J, L}^{(k)}$

These are not yet the final variables we shall use, since the simultaneous occurrence of $h_{\{i, j\}}^{(k)}$ and $h_{\left\{j, i^{\prime}\right\}}^{(k)}$ does not imply a true connection between $D_{J}^{(k)}(i)$ and $D_{J}^{(k)}\left(i^{\prime}\right)$. However, let us first show that when $J \rightarrow \infty$, the bond events $h_{b}^{(k)}$ (which of course depend on $L$ and $J$ ) are driven towards a trivial fixed point.

Proposition 4.6. Let $q>\hat{p}_{c}^{k}$. Then $\tilde{\mu}_{q}\left(h_{b}^{(k)}\right)$ can be made arbitrarily close to one by choosing $J$ sufficiently large.

Proof. Let $\varepsilon>0$. Define $\Delta=\frac{1}{3}\left(q-\hat{p}_{c}^{k}\right)>0$ and choose $N$ so large that $\left(1-\Delta^{k}\right)^{(N / 4 k)}<\frac{1}{2} \varepsilon$.

Let $i, j \in \mathbb{V}^{*}$ be nearest neighbor sites of $\mathbb{V}^{*}$. Consider the diamonds $D_{J}^{(k)}(i)$ and $D_{J}^{(k)}(j)$. Let $s_{J, L}^{(k)}$ be the event discussed in Proposition 4.5 for the diamond $D_{J}^{(k)}(i)$, and denote by $\tilde{t}_{J, L}^{(k)}$ the analogous event of a connection between $E_{J, 3}^{(k)}(j)$ and the bottom of $\Lambda_{L}^{(k)}(\mathrm{i})$. These events are shown in Fig. 19. 
It is clear that $\tilde{t}_{J, L}^{(k)}$ is simply a reflection (through the plane $P$ in Fig. 19) of the event $t_{J, L}^{(k)}$ for a diamond $D_{J}^{(k)}(j)$. Hence $\tilde{\mu}_{q}\left(s_{J, L}^{(k)}\right)=\tilde{\mu}_{q}\left(\tilde{t}_{J, L}^{(k)}\right)$.

By Proposition 4.5 , we can choose $J$ large enough so that $\tilde{\mu}_{q-2 \Delta}\left(s_{J, L}^{(k)}\right) \geqq 1$ $-\frac{1}{4} \varepsilon \Delta^{N}$. Thus

$$
\tilde{\mu}_{q-2 \Delta}\left(s_{J, L}^{(k)} \cap \tilde{t}_{J, L}^{(k)}\right) \geqq 1-\frac{1}{2} \varepsilon \Delta^{N} .
$$

Then, by Lemma 4.2(b), we have

$$
\tilde{\mu}_{q-\Delta}\left[I^{N}\left(s_{J, L}^{(k)} \cap \tilde{t}_{J, L}^{(k)}\right)\right] \geqq 1-\frac{1}{2} \varepsilon .
$$

We claim that any configuration $\underline{n} \in I^{N}\left(s_{J, L}^{(k)} \cap \tilde{t}_{J, L}^{(k)}\right)$ has at least $N / 4 k$ points at which a path emanating from $D_{J}^{(k)}(i)$ overlaps a path emanating from $D_{J}^{(k)}(j)$ within the box $\Lambda_{L}^{(k)}(i)$. (Recall that two paths are said to overlap if their projections intersect.) Suppose, in fact, that in a given configuration $\underline{n} \in \Omega$, there are only $M<N / 4 k$ overlap points. To each point of overlap there corresponds a line of $k$ sites of $\mathbb{L}^{* k}$. Consider the $4 k$ bonds parallel to the $x y$ plane in the coboundaries of these $k$ sites. If we change $\underline{n}$ so that these $4 k$ bonds are not occupied, then the $k$ sites are effectively isolated from the rest of $\mathbb{L}^{* k}$. (In the dual language, this amounts to surrounding these sites by a "chimney" of occupied plaquettes.) Thus, if we alter the configuration $\underline{n}$ by at most $4 k M<N$ bonds, then there are no points of overlap, which means that the altered configuration cannot be in $s_{J, L}^{(k)} \cap \tilde{t}_{J, L}^{(k)}$. Hence $\underline{n} \notin I^{N}\left(s_{J, L}^{(k)} \cap \tilde{t}_{J, L}^{(k)}\right)$.

Next suppose $\underline{n} \in s_{J, L}^{(k)} \cap \tilde{t}_{J, L}^{(k)}$, so that there is at least one point where projections of the paths intersect. If, in addition, all bonds parallel to the $z$ axis at such a point of overlap are occupied, it is clear that the event $h_{\{i, j\}}^{(k)}$ occurs in the configuration $\underline{n}$. In light of this, and of the above observation on the minimum number of overlap points for configurations in $I^{N}\left(s_{J, L}^{(k)} \cap \tilde{t}_{J, L}^{(k)}\right)$, one can show by reasoning along the lines of Lemma 4.2 that

$$
\begin{aligned}
\tilde{\mu}_{q}\left(h_{\{i, j\}}^{(k)}\right) & \geqq\left[1-\left(1-\Delta^{k}\right)^{(N / 4 k)}\right] \tilde{\mu}_{q-\Delta}\left[I^{N}\left(s_{J, L}^{(k)} \cap \tilde{t}_{J, L}^{(k)}\right)\right] \\
& \geqq\left[1-\left(1-\Delta^{k}\right)^{(N / 4 k)}\right]\left[1-\frac{1}{2} \varepsilon\right] \\
& \geqq 1-\varepsilon . \quad \square
\end{aligned}
$$

As remarked above, the events $h_{b}^{(k)}$ do not have the transitivity property which is essential for indicators of connections on the underlying lattice $\mathbb{L}^{* k}$. It is important, therefore, to notice that in the previous proposition only $J$ is required to be large - with no constraints on $L$, as long as $L>J$. We shall use this freedom to chose $L$ even larger, in the construction of renormalized bond events which do have the desired connectivity properties.

Definition. For $i \in \mathbb{V}^{*}$ we denote by $U^{(k)}(i)$ the event

$$
\begin{aligned}
U^{(k)}(i)=\{ & \frac{n \in \Omega \mid \text { all paths of occupied bonds which connect }}{\partial D_{J}^{(k)}(i) \text { with } \partial D_{L}^{(k)}(i) \text { are connected to each other }} \\
& \text { within } \left.D_{L}^{(k)}(i)\right\} .
\end{aligned}
$$

Remark. The event $U^{(k)}(i)$ is not monotone in the sense of FKG, since it can be destroyed by the addition of bonds. 
Proposition 4.7. Let $q>\hat{p}_{c}^{k}$. Then, for each fixed $J$,

$$
\lim _{L \rightarrow \infty} \tilde{\mu}_{q}\left(U^{(k)}(i)\right)=1 \text {. }
$$

Proof. This is essentially a corollary to the proof of uniqueness in Theorem 4.4 [since for $J$ fixed, there can only be a finite number of disjoint connections between $\partial D_{J}^{(k)}(i)$ and $\left.\partial D_{L}^{(k)}(i)\right]$.

Let us finally introduce the bond events which have all the desired properties.

Definition. Let $i, j \in \mathbb{V}^{*}$ be a pair of neighboring sites of $\mathbb{V}^{*}$ (with $|i-j|=\sqrt{2}$ ), and $b=\{i, j\}$. The renormalized bond event $B_{b}$ is defined as follows:

$$
B_{b}=U^{(k)}(i) \cap h_{b}^{(k)} \cap U^{(k)}(j) .
$$

It should be observed that if $B_{\{i, j\}}$ and $B_{\left\{j, i^{\prime}\right\}}$ both occur, then the diamond $D_{J}^{(k)}(i)$ is indeed connected to $D_{J}^{(k)}\left(i^{\prime}\right)$, since both events contain $U^{(k)}(j)$.

We supress in our notation the obvious dependence of $B_{b}$ on $k, L$ and $J$.

Proposition 4.8. For each $q>\hat{p}_{C}^{(k)}$,

$$
\lim _{\substack{L, J \rightarrow \infty \\(L>J)}} \tilde{\mu}_{q}\left(B_{b}^{(k)}\right)=1 .
$$

Proof. This follows immediately from Propositions 4.6 and 4.7.

Summary. The construction of the block bond variables is now complete. Following are the main features of the resulting bond system.

1) First, by Proposition 4.8 , the lengths $J$ and $L$ may be chosen so that the bond events $B_{b}$ occur with probability arbitrarily close to one. Thus, by going to large length scales, we have effectively mapped a system of bonds on $\mathbb{L}^{* k}$ which is near the critical regime into a new bond system on $\mathbb{V}^{*}$ which is controlled by the trivial high density behavior.

2) A novel property of the block bond variables is that despite the fact that they are defined on a coarse scale, the existence of a connected path of "occupied" bonds on $\mathbb{V}^{*}$ implies that there is a corresponding path of bonds on the underlying lattice $\mathbb{L}^{* k}$ which are connected in the "microscopic" sense.

3) Although the bonds of $\mathbb{V}^{*}$ (i.e. the block bonds of $\mathbb{L}^{* k}$ ) are not independently distributed, this lack of independence is only a local effect. Each bond event $B_{b}$ is correlated only with the bonds with which it shares a vertex, and is independent of all the other bond events.

The feature 2) discussed above permits us to reduce the proof of Proposition 4.1 to a simple property of the renormalized bond system. To formulate it, we denote by $\mathbb{V}$ the dual lattice of $\mathbb{V}^{*}$. That is - the sites of $\mathbb{V}$ are the centers of the elementary cells of $\mathbb{V}^{*}, \mathbb{V}$ being isomorphic to $\mathbb{Z}^{2}$ and a translate of $\mathbb{V}^{*}$. For a pair of sites $i, j \in \mathbb{V}$, we denote by $\Gamma(i, j)$ the event that there is a closed path of bonds in $\mathbb{V}^{*}$ which surrounds $i$, separating it from $j$, such that for each of the bonds, the event $B_{b}$ occurs. We now claim that to prove Theorem 4.1, it suffices to show that the following is true. 
Lemma 4.9. If $q>\hat{p}_{c}^{k}$, then for some suitably large $J$ and $L$ there is a positive constant $\kappa=\kappa(q, k, J, L)>0$ such that for each pair of sites $i, j \in \mathbb{V}$,

$$
\tilde{\mu}_{q}[\Gamma(i, j)] \geqq 1-\exp [-\kappa|i-j|] .
$$

Reduction of Theorem 4.1 to Lemma 4.9. The event $\beta^{(k)}\left(x_{1}, x_{2}\right)$ which is discussed in Theorem 4.1 is the existence of a bridge of occupied plaquettes between the two linear segments which are formed by the pins $d_{x_{1}, 0}^{(k)}$ and $d_{x_{2}, 0}^{(k)}$. Such an event is not compatible with the existence of a closed loop of occupied dual bonds which encircle one of the two pins, but not the other. It follows that if $x_{1}, x_{2} \in 2 L \mathbb{Z}$ then:

$$
\tilde{\mu}_{q}\left[\beta^{(k)}\left(x_{1}, x_{2}\right)\right] \leqq 1-\tilde{\mu}_{q}[\Gamma(i, j)],
$$

where $i, j \in \mathbb{V}$ are the lattice sites of $\mathbb{V}$ whose Euclidean coordinates in $\mathbb{R}^{2}$ are $i=\left(\frac{x_{1}}{\sqrt{2} L}, 0\right), j=\left(\frac{x_{2}}{\sqrt{2} L}, 0\right)$.

The claimed bound (4.43) implies, therefore, that (4.2) is satisfied, with $m=\sqrt{2} L \kappa$, whenever $\left(x_{1}-x_{2}\right)$ is a multiple of $2 L$. It then follows by an argument which is strictly analogous to the proof of Proposition 2.5, that (4.43) holds for all $x_{1}$ and $x_{2}$, with the same value of $m$.

Let us now consider Lemma 4.9. It is a statement about the existence of closed loops of occupied bonds which separate two given sites, in a system of random bonds whose density can be made arbitrarily close to one. The bound (4.43) which is claimed there can be proved by a number of methods, which include:

1) A Peierls-type argument, with suitable attention to the lack of independence. This difficulty can be circumvented by considering a sublattice, as was done in a similar situation in [5].

2) An inequality which shows that the function $1-\tilde{\mu}_{q}(\Gamma(i, j))$ is, in a certain sense, "subharmonic," and thus decays exponentially. This path will be followed in Sect. 4ii).

3) An argument in which one directly considers the scaling behavior of certain bond events. It can be shown that if the bond system is sufficiently close to the trivial fixed point, the scaling is exponential. We use such an argument to provide a second proof of Lemma 4.9 in Sect. 4iii).

\section{ii) A High Density Argument}

In this subsection, we provide a proof of Lemma 4.9 by a simple "subharmonicity" inequality. The inequality is analogous to the Griffith's third inequality, which has been used by Krinski and Emery [11] to prove exponential decay of correlations in Ising ferromagnets. In that context, the basic inequality was extended and the method of its application has been improved in the works of Simon [12] and Lieb [13].

First, let us denote for $i, j \in \mathbb{V}$

$$
\tau_{i, j}=1-\tilde{\mu}_{q}(\Gamma(i, j)) .
$$

In order to describe $\tau_{i, j}$ in simple terms it is convenient to associate with each configuration of the bond events $B_{b}$, where $b$ are the bonds of $\mathbb{V}^{*}$, a set of 

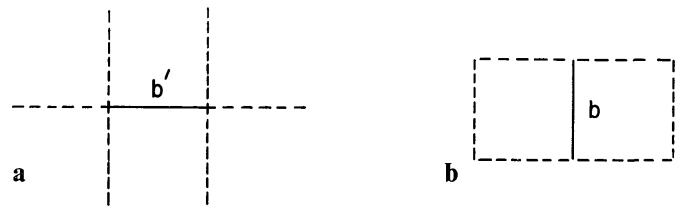

Fig. 20. a Dependent bonds on $\mathbb{V}^{*}$. b Dependent bonds on $\mathbb{V}$

complementary events $\bar{B}_{b}$ on the bonds of the lattice $\mathbb{V}$ (which is dual to $\mathbb{V}^{*}$ ). Thus $\bar{B}_{b}$ occurs, for $b$ on $\mathbb{V}$, if the event $B_{b^{\prime}}$ does not occur for the bond $\mathrm{b}^{\prime}$ of $\mathbb{V}^{*}$ which is dual to $b$. Under the conditions which are referred to in Lemma 4.9, the probability of $\bar{B}$ is very small.

It is easy to see that for $i, j \in \mathbb{V}, \tau_{i, j}$ is the probability that the site $i$ is connected to either $j$, or "infinity," by a path of bonds (of $\mathbb{V}$ ) for which the events $\bar{B}$ occur.

Let us now determine the degree of interdependence of the bond events $\bar{B}$ (on the lattice $\mathbb{V}$ ). First we recall that a bond event $B_{b^{\prime}}, b^{\prime}$ on $\mathbb{V}^{*}$, is independent of all the other bond events, except those which are associated with the six bonds which form the coboundary of $b^{\prime}$ (see Fig. 20a).

By duality, the bond event $\bar{B}_{b}$ is correlated only with those events which are associated with the six bonds of $\mathbb{V}$ which form the boundary of the two "plaquettes" which share $b$ (see Fig. 20b).

It is clear that Lemma 4.9 is equivalent to the statement:

Lemma 4.9'. Under the conditions of Lemma 4.9, there exists a constant $C>0$ such that, for all $i, j \in \mathbb{V}$

$$
\tau_{i, j} \leqq \exp \left[-C\|i-j\|_{\infty}\right] .
$$

Here $\|i-j\|_{\infty}$ denotes the lattice $L^{\infty}$ norm of $i-j$; that is, if $i=\left(i_{1}, i_{2}\right)$ and $j=\left(j_{1}, j_{2}\right)$, then

$$
\|i-j\|_{\infty}=\max \left\{\left|i_{1}-j_{1}\right|,\left|i_{2}-j_{2}\right|\right\} .
$$

In particular $\|i-j\|_{\infty} \geqq|i-j| / \sqrt{2}$.

Our proof is based on the following inequality:

Proposition 4.10. Let $\bar{p}$ denote the probability of a $\bar{B}$ bond event on $\mathbb{V}$. Then, for every $i, j \in \mathbb{V}, i \neq j$,

$$
\tau_{i, j} \leqq 32 \bar{p} \sum_{\substack{l \\\|i-l\|_{\infty}=1}} W(l) \tau_{l, j},
$$

where $W(l)$ are fixed weights which satisfy

$$
\sum_{\substack{l \\\|i-l\|_{\infty}=1}} W(l)=1 .
$$

Proof. $\tau_{i, j}$ is the probability that $i$ is connected to $j$, or to infinity, by a path of bonds on which $\bar{B}$ occurs. Whenever such an event occurs, for $i \neq j$, then: 


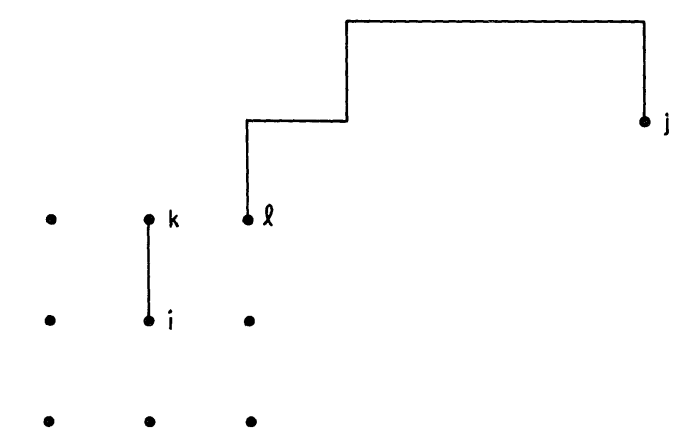

Fig. 21. The event $\bar{B}_{\{i, k\}} \cap T_{l, j}^{\prime}$

1) The event $\bar{B}_{\{i, k\}}$ occurs for at least one of the nearest-neighbors, $k \in \mathbb{V}$, of $i$ (with $|k-i|=1$ ).

2) There is a site $l$, with $\|l-i\|_{\infty}=1$, which is connected (in the above sense) to either $j$ or infinity, by a path which avoids all the other points $l^{\prime}$ with $\left\|l^{\prime}-i\right\|_{\infty}=1$. We denote such an event by $T_{l, j}^{\prime}$ (Fig. 21).

Thus:

$$
\tau_{i, j} \leqq \sum_{\substack{k, l \\|k-i|=\|l-i\|_{\infty}=1}} \tilde{\mu}_{q}\left(\bar{B}_{\{i, k\}} \cap T_{l, j}^{\prime}\right) .
$$

However, due to observations made above, $\bar{B}_{\{i, k\}}$ and $T_{l, j}^{\prime}$ are independent events, and furthermore $\tilde{\mu}_{q}\left(T_{l, j}^{\prime}\right) \leqq \tau_{l, j^{\prime}}$. Therefore (4.48) implies

$$
\tau_{i, j} \leqq \sum_{\substack{k, l \\|k-i|=\|l-i\|_{\infty}=1}} \tilde{\mu}_{q}\left(\bar{B}_{\{i, k\}}\right) \tau_{l, j}=4 \bar{p} \sum_{\|l-i\|_{\infty}=1} \tau_{l, j} .
$$

Letting $W(l)=\frac{1}{8}$, we obtain the desired result (4.46).

Proof of Lemma 4.9. The inequality (4.46) may be iterated by applying it to $\tau_{l, j}$, as long as $l \neq j$. Each iteration adds a factor of $(32 \bar{p})$ which multiplies a normalized average of the function $\tau_{, j}$, which is everywhere bounded by 1 . Since for $i \neq j(4.46)$ may be safely iterated $\|i-j\|_{\infty}$ times, we obtain

$$
\tau_{i, j} \leqq(32 \bar{p})^{\|i-j\|_{\infty}} \text {. }
$$

Choosing $L$ and $J$ large enough so that $32 \bar{p}<1$, we have a proof of Lemma 4.9' (and thus Lemma 4.9), with $C=-\log (32 \bar{p})$.

Remarks. 1) A slight refinement of the above argument permits us to replace the factor $(32 \bar{p})$ in $(4.50)$, and in the derived value of $C$, by $(20 \bar{p})$.

2) As already mentioned, (4.46) is somewhat analogous to the Griffith's third inequality for ferromagnets. The simple iteration argument which we used to prove Lemma $4.9^{\prime}$ is similar to one used by Simon [12]. The more general SimonLieb inequality (for Ising ferromagnets) also has a counterpart in percolation models, which has been noted by a number of people including B. SouillardF. Delyon and A. Sokal, and is described in the appendix of [14]. 


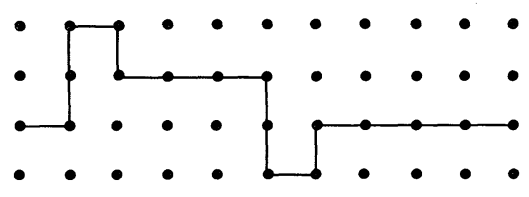

Fig. 22. The event $R(4,11)$

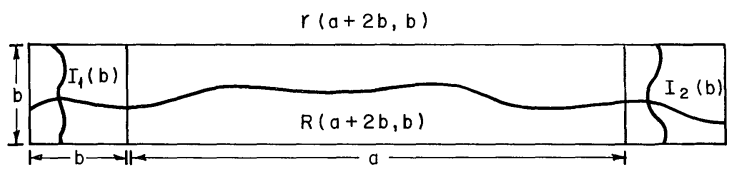

Fig. 23. The event $\mathbb{B}(a, b)$

\section{iii) A Rescaling Argument}

We now offer a second proof of Lemma 4.9 in which the bond lattice $\mathbb{V}^{*}$ is further rescaled. This proof is the (destructive) counterpart of the proof of length law given in Sect. 3ii). The latter uses rescaled plaquette variables to construct the event $W_{\gamma}$; here, we shall use rescaled bond variables to destroy the event $W_{\gamma}$.

We first establish some preliminary results which parallel those of Sect. 3ii). As in Sect. 4ii), we fix $k$ and present two-dimensional arguments in which we omit all reference to the superscript $(k)$.

Definition. Let $r(a, b)$ denote an $a \times b$ rectangle of vertices of the renormalized lattice $\mathbb{V}^{*}$

$$
r(a, b)=\left\{\left(i_{1}, i_{2}\right) \in \mathbb{V}^{*} \mid 0<i_{1} \leqq a, 0<i_{2} \leqq b\right\} .
$$

Here $i_{1}$ and $i_{2}$ label coordinates along axes at $45^{\circ}$ to those of the lattice $\mathbb{L}^{* k}$ (and hence along the direction of the bonds of $\mathbb{V}^{*}$ ).

Let $R(a, b)$ denote the event of a left-right crossing of $r(a, b)$ by renormalized bonds $B$ with both vertices in the set $r(a, b)$ (see Fig. 22).

We note, without proof, the following trivial observation.

Proposition 4.11. For every integer $n \geqq 1$

$$
\tilde{\mu}_{q}[R(a, b)] \geqq \sqrt[n]{\tilde{\mu}_{q}[R(n a, b)]} .
$$

Next, we consider events in rectangles of the form $r(a+2 b, b)$. Each such rectangle is regarded as consisting of an inner rectangle, which is a translate of $r(a, b)$, and two outer squares, which are translates of $r(b, b)$. We refer to the outer squares as the left and right ends of $r(a+2 b, b)$.

Definition. Let $I_{1}(b)$ be the event of a top-bottom crossing of the rectangle $r(a+2 b, b)$ which lies entirely in the left end. Similarly, $I_{2}(b)$ denotes the analogous event in the right end. We note that $I_{1}(b)$ and $I_{2}(b)$ are translates and rotations of $R(b, b)$. We define the rescaled bond event (see Fig. 23) 


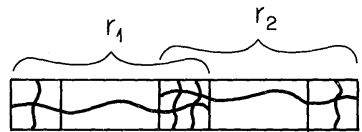

a

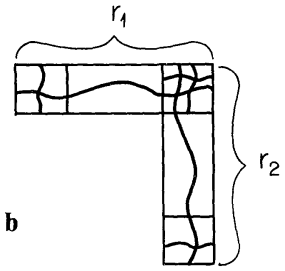

Fig. 24a and b. Connection of bond events

$$
\mathbb{B}(a, b)=R(a+2 b, b) \cap\left[\bigcap_{i=1}^{2} I_{i}(b)\right] .
$$

Remark. It is observed that the rescaled bond events $\mathbb{B}(a, b)$ have the required connectivity properties of bonds. In particular, if two rectangles of the same dimension, $r_{1}$ and $r_{2}$, are placed side by side so that adjacent ends overlap, and if the rescaled bond event $\mathbb{B}$ occurs in both rectangles, then there is a left-right crossing of the larger rectangle $r_{1} \cup r_{2}$ (see Fig. 24a).

If $r_{1}$ and $r_{2}$ are placed perpendicular to one another so that they have an end region in common, then the occurrence of rescaled bond events in both rectangles guarantees the existence of a continuous crossing from the left end of $r_{1}$ to the bottom of $r_{2}$ (see Fig. 24b).

As is the case for rescaled plaquette events, bond events of different length scales also have desirable connectivity properties. However, this feature is not used in the proof of Lemma 4.9 .

The bond events $\mathbb{B}(a, b)$ exhibits the following behavior under rescaling.

Lemma 4.12. If, for some $a$ and $b$,

$$
\tilde{\mu}_{q}[\mathbb{B}(a, b)] \geqq 1-\beta \lambda,
$$

with $\beta=1 / 18$ and some $\lambda<1$, then

$$
\tilde{\mu}_{q}[\mathrm{~B}(3 a, 2 b)] \geqq 1-\beta \lambda^{2} .
$$

Proof. We place three translates of $r(a+2 b, b)$ side by side with ends overlapping to form the longer rectangle $r(3 a+4 b, b)$.

If bond events $\mathbb{B}(a, b)$ occur in all three rectangles (see Fig. 25), then the event $R(3 a+4 b, b)$ occurs.

This gives us

$$
\tilde{\mu}_{q}[R(3 a+4 b, b)] \geqq 1-3 \beta \lambda .
$$

Next we observe that the rectangle $r(3 a+4 b, 2 b)$ consists of two translates of $r(3 a+4 b, b)$ which have no vertices of $\mathbb{V}^{*}$ in common. Since the aforementioned dependence of the bonds of $\mathbb{V}^{*}$ only occurs among bonds which share a vertex, the events of left-right crossings in the upper and lower halves of $r(3 a+4 b, 2 b)$ are completely independent. We obtain the estimate

$$
\tilde{\mu}_{q}[R(3 a+4 b, 2 b)] \geqq 1-(3 \beta \lambda)^{2} .
$$




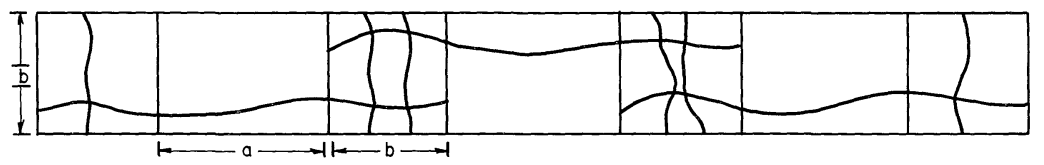

Fig. 25. Construction of $R(3 a+4 b, b)$ from three translates of $\mathbb{B}(a, b)$

By translation invariance, $\tilde{\mu}_{q}\left[I_{i}(2 b)\right]=\tilde{\mu}_{q}[R(2 b, 2 b)]$. Then, since the events $I_{1}(2 b)$ and $I_{2}(2 b)$ occur in disjoint regions,

$$
\tilde{\mu}_{q}\left[\bigcap_{i=1}^{2} I_{i}(2 b)\right]=\tilde{\mu}_{q}^{2}[R(2 b, 2 b)] \geqq \tilde{\mu}_{q}[R(3 a+4 b, 2 b)] .
$$

The last step in (4.58) follows from Proposition 4.11.

Thus, by Eqs. (4.57) and (4.58), we have

$$
\tilde{\mu}_{q}[\mathbb{B}(3 a, 2 b)] \geqq 1-2(3 \beta \lambda)^{2}=1-\beta \lambda^{2} .
$$

Remark. Iterating the above inequality $l$ times, we obtain

$$
\tilde{\mu}_{q}\left[\mathrm{~B}\left(3^{l} a, 2^{l} b\right)\right] \geqq 1-\beta \lambda^{2^{l}} .
$$

Just as plaquette events of many length scales were used in the proof of perimeter law, we shall use bond events of many length scales to prove Lemma 4.9. However, it is also interesting to consider a lattice of block bonds $\mathbb{B}\left(3^{l} a, 2^{l} b\right)$ of uniform scale. As $l$ increase, the common region of adjacent bonds, which should be viewed as a vertex on the rescaled lattice, becomes an increasingly smaller part of a given bond event. Thus the interdependence of bonds emanating from the same vertex becomes progressively weaker as the lattice is further rescaled.

Proof of Lemma 4.9. Let $i, j \in \mathbb{V}$. By the equivalence of the norms $|i-j|$ and $\|i-j\|_{\infty}$, it suffices to prove that there exists a constant $C>0$ such that

$$
\Gamma(i, j) \geqq 1-\exp \left[-C\|i-j\|_{\infty}\right] .
$$

By Proposition 4.8, there are length scales $J(k)$ and $L(k)$ such that $\tilde{\mu}_{q}(B)>31 / 32$.

Taking $\mathbb{B}(1,1)$ as the first rescaled bond event, we have $17 / 18<\tilde{\mu}_{q}[\mathbb{B}(1,1)] \equiv 1$ $-\beta e^{-C}$ with $\beta=1 / 18$ and $C>0$. Hence, by Lemma 4.12 , the probability of the $l^{\text {th }}$ rescaled bond event is

$$
\tilde{\mu}_{q}\left[\mathrm{~B}\left(3^{l}, 2^{l}\right)\right] \geqq 1-\beta e^{-2^{l} \mathrm{C}} .
$$

Let $G(l)$ be a fundamental ringlet of four bond events $\mathbb{B}\left(3^{l}, 2^{l}\right)$ (see Fig. 26).

We have

$$
\tilde{\mu}_{q}[G(l)] \geqq 1-4 \beta e^{-2^{l} C}>1-e^{-2^{l} C} .
$$

Notice that if a ringlet $G(l)$ surrounds the vertex $i$ (and not the vertex $j$ ), then the event $\Gamma(i, j)$ occurs. The number and scales of the ringlets that can be used to efficiently separate $i$ from $j$ is determined by the binary expansion of $\|i-j\|_{\infty}$, i.e.

$$
\|i-j\|_{\infty}=2^{l_{1}}+2^{l_{2}}+\ldots+2^{l_{n}} .
$$




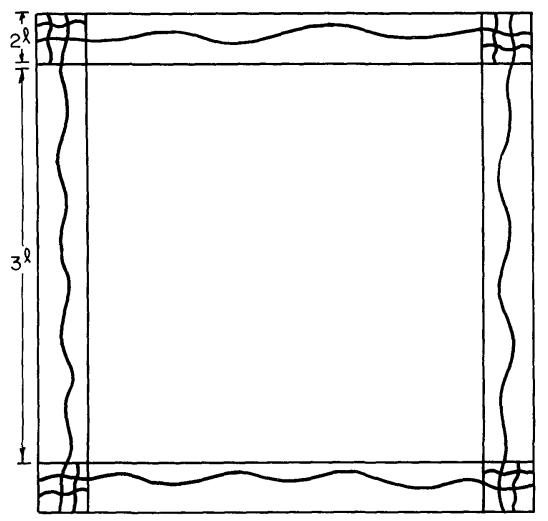

Fig. 26. The event $G(l)$

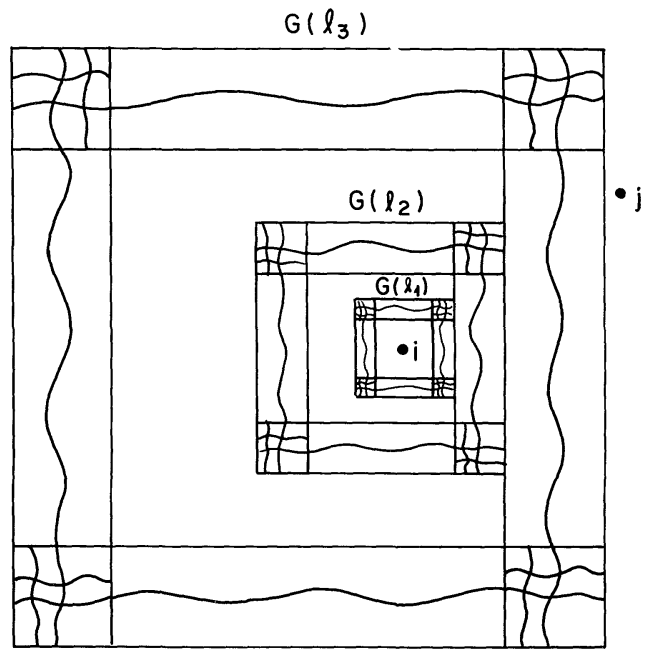

Fig. 27. Ringlets separating $i$ from $j$

Denoting the annulus in which the event $G(l)$ may occur by $A(l)$, it is clear that disjoint translates of all annuli $A\left(l_{i}\right)$ with $i \leqq n$ may be inserted between $i$ and $j$ (see Fig. 27).

The probability of the occurrence of at least one of the events $G\left(l_{i}\right)$ is given by

$$
\begin{aligned}
\tilde{\mu}_{q}\left[\bigcap_{i=1}^{n} G\left(l_{i}\right)\right] & \geqq 1-\exp \left[-C \sum_{i=1}^{n} 2^{l_{i}}\right] \\
& =1-\exp \left[-C\|i-j\|_{\infty}\right],
\end{aligned}
$$

which is the lower bound for $\Gamma(i, j)$. 


\section{Summary of Results and Open Problems in Three Dimensions}

In this section, we briefly review our principal results on the behavior of $\left\langle W_{\gamma}\right\rangle_{p}$ in three dimensions and discuss our conjecture on the equality of critical points for percolation. We also contrast the critical behavior of $\left\langle W_{\gamma}\right\rangle_{p}$ with that of $\left\langle W_{\gamma}^{0}\right\rangle_{p}$ and $\left\langle W_{\gamma}^{S}\right\rangle_{p}$.

First, however, we would like to point out that perhaps the most interesting question is the existence, and the properties of the continuum limit for loop variables. An essential step for the analysis of the scaling limit is to determine whether the surface tension, $\alpha(p)$, vanishes when $p \rightarrow p_{c}$. (Our guess is that it does.)

Let us now recall the definitions of the two critical bond probabilities which characterize the surface dominated and dual percolation regimes. The former is $\pi_{c}$, the point at which the expected size of bond clusters becomes infinite and the latter is $\varrho_{c}$, the limiting value of the quadrant bond percolation threshold for layers of increasing thickness.

We have shown that there exist constants $0<c(p), \alpha(p)<\infty$ such that

$$
\left\langle W_{\gamma}\right\rangle_{p} \sim \begin{cases}\exp [-c(p) \operatorname{Per}(\gamma)] ; & p>1-\pi_{c} \\ \exp [-\alpha(p) \operatorname{Area}(\gamma)] ; & p<1-\hat{\varrho}_{c} .\end{cases}
$$

The perimeter law behavior, which holds for planar loops, follows from Proposition 2.3, Theorem 3.2 and the discussion in Sect.3iii). The area law behavior, which we proved for rectangular loops, is a consequence of Propositions 2.3-2.5 and the corollary to Theorem 4.1.

A question which remains to be addressed, in the analysis of the model's phase structure, is our conjecture on the equality of critical points for three-dimensional percolation:

$$
\pi_{c}=p_{c}=\varrho_{c}=\hat{\varrho}_{c} .
$$

Let us clarify the relationship between Eq. (5.2) and other long-standing conjectures in percolation theory.

The equality $\pi_{c}=p_{c}$, expected in any dimension, is a very natural conjecture. The same equality has been proved in two dimensions $[4,5,7]$ and is usually assumed true in non-mathematical papers.

The conjectured equality $p_{c}=\varrho_{c}$ requires more discussion. If this is correct, then whenever bonds percolate on the full lattice, there is also a sufficiently thick finite layer in which they percolate. We should remark that the analog of $p_{c}=\varrho_{c}$ concerning percolation in strips is obviously false in two dimensions. However, this is clearly a consequence of the fact that one is the lower critical dimension for percolation and has no bearing on the three-dimensional conjecture. As we shall show below, the equality $p_{c}=\varrho_{c}$ is in fact related to the widely believed conjecture that the infinite cluster is unique in three dimensions.

The final equality, $\varrho_{c}=\hat{\varrho}_{c}$, which says that the limiting value of the bond percolation threshold for layers is the same as that for quadrant layers, is also quite reasonable. In two dimensions, which is simply a layer with $k=1$, the analogous equality (i.e., $p_{c}^{1}=\hat{p}_{c}^{1}$ ) is well known, and we expect that the limiting percolation thresholds also have this property. 
Given the results of Sects. 2-4, let us consider the implications of the conjectured equality (5.2). First, Eq. (5.1) would then imply that $\left\langle W_{\gamma}\right\rangle_{p}$ undergoes a sharp transition from area law to perimeter law behavior at the critical point $p=1$ $-\pi_{c}=1-\hat{\varrho}_{c}$.

We claim that this conjecture also implies that the three-dimensional bond connectivity function, $\tau_{i, j}(p)$, exhibits a transition at the same critical point as $\left\langle W_{\gamma}\right\rangle_{p}$. This follows from the asymptotic behavior:

$$
\tau_{i, j} \sim \begin{cases}\exp [-m(p)|i-j|] ; & p>1-\pi_{c} \\ c(p) ; & p<1-\hat{\varrho}_{c} .\end{cases}
$$

The exponential decay for $p>1-\pi_{c}$ has been shown by Kesten [10] (see also Aizenman and Newman [14]). The constant behavior for $p<1-\hat{\varrho}_{c}$ is easily proved from the results of Sect. 4i). The FKG inequality implies that $\tau_{i, j}$ is bounded below by the square of the probability of events of the form $\Xi^{(k)}$, which, by Proposition 4.2 , do not decay to zero for $p<1-\hat{\varrho}_{c}^{k}$.

We may regard the area law for $\left\langle W_{\gamma}\right\rangle_{p}$ as a two-volume law and the perimeter law as the corresponding boundary law. Similarly, the exponential behavior of $\tau_{i, j}$ should be viewed as a one-volume law, with the asymptotically constant behavior as its corresponding boundary law. If our conjecture is correct, then $\left\langle W_{\gamma}\right\rangle_{p}$ exhibits volume behavior whenever $\tau_{i, j}$ exhibits boundary behavior and vice versa. In other words, dual objects in three dimensions are always in opposite phases. The $d$-dimensional version of this phenomenon is discussed in Sect. 6.

The features discussed above are closely related to uniqueness of the infinite cluster. In Sect. 4 i), we proved that, for $p<1-\hat{p}_{c}^{k}$, the infinite cluster in the $k$-layer $\mathbb{L}^{* k}$ is unique (Theorem 4.4). It is easy to extend this result to uniqueness of the infinite cluster on the full three-dimensional lattice $\mathbb{L}^{*}$. To see this, we follow exactly the proof of Theorem 4.4 , replacing the boxes $\Delta_{N}$ by cubes $\Delta_{N}^{\prime}$, for which the probability of the corresponding events $A_{N}^{\prime}$ is bounded above by $1-[a(q)]^{4}$ $<1$.

The above result shows that the equality $p_{c}=\varrho_{c}=\hat{\varrho}_{c}$ implies the long-standing conjecture that, at least in three dimensions, the infinite cluster is always unique.

Finally, let us discuss the quantities $\left\langle W_{\gamma}^{0}\right\rangle_{p}$ and $\left\langle W_{\gamma}^{S}\right\rangle_{p}$, introduced in Sect. 2. Since $W_{\gamma}^{0} \subset W_{\gamma}$, we have $\left\langle W_{\gamma}^{0}\right\rangle_{p} \leqq\left\langle W_{\gamma}\right\rangle_{p}$. Hence whenever $\left\langle W_{\gamma}\right\rangle_{p}$ obeys an area law, so does $\left\langle W_{\gamma}^{0}\right\rangle_{p}$. Moreover, in Sect. 3, we explicitly showed that $\left\langle W_{\gamma}^{0}\right\rangle_{p}$ obeys a perimeter law for $p<1-\pi_{c}$ by constructing manifestly orientable surfaces. Thus, if our conjecture $\pi_{c}=\varrho_{c}$ is correct, $\left\langle W_{\gamma}\right\rangle_{p}$ and $\left\langle W_{\gamma}^{0}\right\rangle_{p}$ must have the same transition point.

However, we expect that $\left\langle W_{\gamma}^{S}\right\rangle_{p}$ should have a transition from area to perimeter law at a strictly larger $p$ than the critical value for $\left\langle W_{\gamma}\right\rangle_{p}$. To see this, note that percolating chains of occupied bonds which interlock $\gamma$ only permit the formation of surfaces with handles (see Fig. 2). Thus when chains percolate, it is likely that $\left\langle W_{\gamma}^{S}\right\rangle_{p}$ obeys an area law. Since connected chains of occupied bonds are more probable than connected paths of occupied bonds, one expects that infinite chain clusters should form at a bond probability strictly smaller than $p_{c}$. Indeed, in two dimensions, it is known that different notions of connectedness (e.g., star vs. nearest neighbor) lead to different percolation thresholds $[15,16]$. 


\section{Higher Dimensional Generalizations}

In this section, we examine higher dimensional analogs of the systems treated previously. First, in Subsect. i), we consider the system of random plaquettes on $\mathbb{Z}^{d}$. In Subsect. ii), we study the percolation of random $r$-cells in $d>r$ dimensions and examine the generalization of the Wilson loop for these systems. In the final subsection, we briefly discuss the possible phase structure of percolation models in $d>3$.

\section{i) Tree Approximation Bounds for Plaquettes on $\mathbb{Z}^{d}$}

Consider a system of plaquettes distributed with uniform density $p$ on the lattice $\mathbb{Z}^{d}$. Given a loop $\gamma$ of edges on $\mathbb{Z}^{d}$, the event $W_{\gamma}^{d}$ is defined as in Eq. (2.3).

We immediately observe that $\left\langle W_{\gamma}^{d}\right\rangle_{p}$ has a priori upper and lower bounds of perimeter and area law behavior for all $d \geqq 2$.

Proposition 6.1. For every rectangular loop $\gamma$ of edges on $\mathbb{Z}^{d}$

$$
\exp [-|\log p| \operatorname{Area}(\gamma)] \leqq\left\langle W_{\gamma}^{d}\right\rangle_{p} \leqq \exp \left[-c_{d}(p) \operatorname{Per}(\gamma)\right],
$$

where $c_{d}(p)=\left|\log \left[1-(1-p)^{2(d-1)}\right]\right|$.

Proof. See Proposition 2.3.

The following theorem shows that, for any $d \geqq 2$, an area law phase exists below some nonzero plaquette density.

Theorem 6.2. For any loop $\gamma$ of edges on $\mathbb{Z}^{d}$

$$
\left\langle W_{\gamma}^{d}\right\rangle_{p} \leqq \exp \left[-\hat{c}_{d}(p) \operatorname{Area}(\gamma)\right],
$$

with $\hat{c}_{d}(p)>0$ whenever $p<\frac{1}{2(d-1)}$.

Proof. Let $e \in \gamma$ be an edge of $\gamma$ and consider the $2(d-1)$ plaquettes in the coboundary of $e$. For each $Q \in \hat{\partial} e$, we define the new loop $\gamma_{Q}=\gamma \Delta \partial \mathrm{Q}$.

Clearly, if the event $W_{\gamma}^{d}$ occurs, then there is at least one $Q \in \hat{\partial} e$ such that (1) $Q$ is occupied, and (2) there is a surface spanning $\gamma_{Q}$ which avoids the plaquette $Q$.

Let the former event be denoted simply by $Q$ and the latter by $\bar{W}_{\gamma_{Q}}^{d}$. Thus

$$
W_{\gamma}^{d}=\bigcup_{Q \in \hat{\partial} e}\left(Q \cap \bar{W}_{\gamma_{Q}}^{d}\right)
$$

so that

$$
\begin{aligned}
\left\langle W_{\gamma}^{d}\right\rangle_{p} & \leqq \sum_{Q \in \hat{\partial} e} \mu_{p}\left(Q \cap \bar{W}_{\gamma_{Q}}^{d}\right) \\
& =\sum_{Q \in \hat{\partial} e} p\left\langle\bar{W}_{\gamma_{Q}}^{d}\right\rangle_{p} \\
& \leqq \sum_{Q \in \hat{\partial} e} p\left\langle W_{\gamma_{Q}}^{d}\right\rangle_{p} .
\end{aligned}
$$

The second step in (6.4) follows from independence of $Q$ and $\bar{W}_{\gamma Q}^{d}$, and the final inequality is obtained by relaxing the restriction that the surface spanning $\gamma_{Q}$ must avoid the plaquette $Q$. 
Whenever $p<\frac{1}{2(d-1)}$, Eq. (6.4) may be rewritten:

$$
\left\langle W_{\gamma}^{d}\right\rangle_{p} \leqq e^{-\hat{c}_{d}(p)}\left[\sum_{Q \in \hat{\partial} e} \frac{1}{2(d-1)}\left\langle W_{\gamma_{Q}}^{d}\right\rangle_{p}\right],
$$

where $e^{-\hat{c}_{d}(p)} \equiv 2(d-1) p<1$. Observe that the term in brackets above is a normalized sum of probabilities.

The above inequality may be iterated by applying it to $\left\langle W_{\gamma_{Q}}^{d}\right\rangle_{p}$, as long as the "loop" $\gamma_{Q}$ (which may consist of several disjoint loops) has at least one plaquette in its minimal area. Thus (6.5) may be safely iterated Area $(\gamma)$ times. Bounding the final normalized sum by one, we obtain

$$
\left\langle W_{\gamma}^{d}\right\rangle_{p} \leqq \exp \left[-\hat{c}_{d}(p) \operatorname{Area}(\gamma)\right] .
$$

Corollary. For any rectangular loop $\gamma$ of edges on $\mathbb{Z}^{d}$

$$
\left\langle W_{\gamma}^{d}\right\rangle_{p} \sim \exp \left[-\alpha_{d}(p) \text { Area }(\gamma)\right],
$$

with $0<\alpha_{d}(p)<\infty$ whenever $p<\frac{1}{2(d-1)}$.

Proof. This follows immediately from Propositions 2.4 and 2.5 and the above estimates.

Next we observe that $\left\langle W_{\gamma}^{d}\right\rangle_{p}$ for $d \geqq 3$ is bounded below by $\left\langle W_{\gamma}^{3}\right\rangle_{p}$, the probability that the loop is spanned in a three-dimensional subspace. By the results of Sect. 3, this implies that $\left\langle W_{\gamma}^{d}\right\rangle_{p}$ has a lower bound of perimeter law behavior whenever $p>1-\pi_{c}$.

The above reasoning in fact proves that for $d \geqq 3$, there is always a perimeter law phase. A stronger result is established in the following theorem.

Theorem 6.3. For any rectangular loop $\gamma$ on $\mathbb{Z}^{d}, d \geqq 3$,

$$
\left\langle W_{\gamma}^{d}\right\rangle_{p} \geqq \exp \left[-c_{d}^{\prime}(p) \operatorname{Per}(\gamma)\right]
$$

with $c_{d}^{\prime}(p)<\infty$ whenever

$$
p>\bar{p}(d) \sim(\text { const }) d^{-1 / 5}
$$

Proof. As remarked above, whenever $p>1-\pi_{c}$, we obtain a lower bound of perimeter law behavior by considering onty those configurations for which $\gamma$ is spanned in some three-dimensional subspace of $\mathbb{Z}^{d}$. We shall improve this estimate by showing that if plaquettes have some density $p$ on $\mathbb{Z}^{d}$, then there is an effective plaquette density greater than $p$ on a three-dimensional subspace of $\mathbb{Z}^{d}$.

For simplicity, let us assume that $d=3 N$ with $N \geqq 2$. Then we may regard $\mathbb{Z}^{d}$ as the product $\left[\mathbb{Z} \times \mathbb{Z}^{N-1}\right]^{3}$, so that to each of the three orthogonal directions in $\mathbb{Z}^{3}$, we associate an extra $N-1$ dimensions.

Let $x, y$, and $z$ denote the axes in the three-dimensional subspace. Focus attention on a single plaquette $Q$ in the $x y$ plane, and denote by $t_{1}, \ldots, t_{N-1}$ axes along the extra directions associated with this plane. 
Either the plaquette $Q$ is occupied or, if not, it may be "effectively occupied" by events which occur in the extra $N-1$ directions. To see this, consider the cube which is directly above $Q$ in the $t_{1}$ direction and for which $Q$ is the bottom face.

Denote by $q_{1}$ the set of five plaquettes which form the five faces, excluding $Q$, of the aforementioned cube. Similarly, consider the cubes above $Q$ in the other $N-2$ directions, $t_{2}, \ldots, t_{N-1}$, and denote by $q_{2}, \ldots, q_{N-1}$ the corresponding sets of five plaquettes.

Let $S$ be any finite collection of plaquettes, excluding $Q$, in the threedimensional subspace. Clearly, for every $i$,

$$
\partial(S \cup Q)=\partial\left(S \cup q_{i}\right) .
$$

Thus an "effective plaquette event," $E_{Q}$, for $Q$, is that either $Q$ is occupied, or if it is not occupied, then in at least one of the $N-1$ orthogonal directions, the five plaquettes in $q_{i}$ are occupied. We have

$$
\mu_{p}\left(E_{Q}\right)=p+(1-p)\left[1-\left(1-p^{5}\right)^{N-1}\right] .
$$

Clearly, the event that the plaquettes in $q_{i}$ are occupied is independent of any events in the three-dimensional subspace.

In order to construct mutually independent events $E_{Q}$ for every plaquette $Q$ in the $x y$ plane, the plane should be viewed as a "checkerboard" of even and odd plaquettes. Then for even plaquettes, the relevant cube events are defined in the positive $t$ directions, while for odd plaquettes, they are defined in the negative $t$ directions.

Similarly, we may use these extra $N-1$ directions to augment the probabilities of plaquettes in all planes parallel to the $x y$ plane.

Finally, the remaining $2(N-1)$ extra dimensions are used to enhance the probabilities of plaquettes parallel to the $x z$ and $y z$ planes.

In this construction, all plaquette events $E_{Q}$ are independent. Thus we have an effective plaquette probability

$$
P_{\text {eff }}=p+(1-p)\left[1-\left(1-p^{5}\right)^{(d-3) / 3}\right]
$$

for a three-dimensional problem. Therefore, whenever $P_{\text {eff }}>1-\pi_{c}$, the system is in the perimeter law phase. It is not difficult to show that this condition is satisfied if

$$
p>\left(3\left|\log \pi_{c}\right|\right)^{1 / 5}(d-3)^{-1 / 5} .
$$

We note that if $d$ is not a multiple of three, the estimate in (6.13) holds with $d$ replaced by the largest multiple of three less than $d$.

\section{ii) Cells of Higher Dimension}

The cubic lattice $\mathbb{Z}^{d}$ contains $d$ elementary objects (excluding sites) which are candidates for stochastic-geometric study.

Let us consider the Bernoulli system of elementary $r$-cells on $\mathbb{Z}^{d}, d \geqq r$. Cells are occupied with homogeneous probability $p$ and vacant with probability $1-p$. Two $r$-cells are said to be connected if they have a boundary in common. (We note that, at least with this definition of connectedness, the problem of sites on $\mathbb{Z}^{d}$ is trivial.)

It is clear that $r$-cell systems at density $p$ are dual to $(d-r)$-cell systems at density $1-p$. 
Although the onset of infinite connected clusters of $r$-cells is a useful characterization of the phase structure in some contexts, such infinite clusters do not necessarily reflect the $r$-dimensionality of their elementary constitutents. As an alternative, we propose that the asymptotic behavior of the generalized connectivity event ( $\tau_{i, j}$ for bonds, $W_{\gamma}$ for plaquettes) may serve as the order parameter which distinguishes high and low density phases of the system.

The connectivity event, $\left(D_{\Gamma}\right)_{r}^{d}$, for $r$-cells in $d$ dimensions is defined as follows. Given a closed (self-avoiding) surface $\Gamma$, composed of $(r-1)$-cells, the event $\left(D_{\Gamma}\right)_{r}^{d}$ is said to occur if there exists a finite collection of occupied $r$-cells on $\mathbb{Z}^{d}$, the boundary of which is $\Gamma$.

We shall say that $\left(D_{\Gamma}\right)_{r}^{d}$ obeys a volume law if

$$
\left.\left\langle\left(D_{\Gamma}\right)_{r}^{d}\right\rangle_{p} \sim \exp [- \text { const }) \operatorname{Vol}(\Gamma)\right],
$$

where $\operatorname{Vol}(\Gamma)$ is the minimum number of $r$-cells whose boundary is $\Gamma$. Similarly, $\left(D_{\Gamma}\right)_{r}^{d}$ is said to obey a boundary law if

$$
\left\langle\left(D_{\Gamma}\right)_{r}^{d}\right\rangle_{p} \sim \exp [- \text { const) } \operatorname{Bnd}(\Gamma)],
$$

where $\operatorname{Bnd}(\Gamma)$ is the number of $(r-1)$-cells in the surface $\Gamma$.

Analogs of all the results which were proved for plaquettes (2-cells) on $\mathbb{Z}^{d}$ in Subsect. i) are easily established for random $r$-cells.

First, we observe that $r$-cells in $r$ dimensions always obey a volume law. Hence, at least in terms of the order parameter $\left\langle\left(D_{\Gamma}\right)_{r}^{d}\right\rangle_{p}$, there are only $d-1$ nontrivial models in $d$ dimensions.

We again obtain a priori upper and lower bounds:

Proposition 6.4. For any hyperrectangular surface $\Gamma$ of $(r-1)$-cells on $\mathbb{Z}^{d}, d \geqq r$

$$
\exp [-|\log p| \operatorname{Vol}(\Gamma)] \leqq\left\langle\left(D_{\Gamma}\right)_{r}^{d}\right\rangle_{p} \leqq \exp \left[-c_{r}^{d}(p) \operatorname{Bnd}(\Gamma)\right],
$$

where $c_{r}^{d}(p) \equiv\left|\log \left[1-(1-p)^{2[d-(r-1)]}\right]\right|$.

Proof. See Propositions 2.3 and 6.1.

Next we note that for any $d \geqq r$, random $r$-cells on $\mathbb{Z}^{d}$ exhibit volume phase behavior below some nonzero density.

Theorem 6.5. For any closed surface $\Gamma$ of $(r-1)$-cells on $\mathbb{Z}^{d}, d \geqq r$,

$$
\left\langle\left(D_{\Gamma}\right)_{r}^{d}\right\rangle_{p} \leqq \exp \left[-\hat{c}_{r}^{d}(p) \operatorname{Vol}(\Gamma)\right]
$$

with $\hat{c}_{r}^{d}(p)>0$ whenever $p<\frac{1}{2[d-(r-1)]}$.

Proof. See Theorem 6.2.

Corollary. For any hyperrectangular surface $\Gamma$ of $(r-1)$-cells on $\mathbb{Z}^{d}, d \geqq r$,

$$
\left\langle\left(D_{\Gamma}\right)_{r}^{d}\right\rangle_{p} \sim \exp \left[-\alpha_{r}^{d}(p) \operatorname{Vol}(\Gamma)\right]
$$

with $0<\alpha_{r}^{d}(p)<\infty$ whenever $p<\frac{1}{2[d-(r-1)]}$. 
The constant $\alpha_{r}^{d}(p)$ is the analog of the surface tension (or string tension).

To obtain estimates on the density above which the system exhibits boundary law behavior, we parallel the strategy employed for plaquettes in Subsect. i). First, we examine the random $r$-cell model in $d=r+1$ dimensions, which has as its dual the bond model in $\mathbb{Z}^{r+1}$. Although we do not know the critical probability for the onset of boundary law in these systems, it is easy to obtain an upper bound on this probability (Proposition 6.6). This bound will then serve as an estimate for the onset of boundary law behavior in all $d \geqq r+1$. Finally, in Theorem 6.7 , we shall employ the method of dimensional enhancement used in Theorem 6.3 to improve this estimate.

Let $\pi_{c}^{d}$ denote the critical bond density at which the expected size of bond clusters on $\mathbb{Z}^{d}$ diverges.

Proposition 6.6. For any hyperrectangular surface $\Gamma$ of $(r-1)$-cells on $\mathbb{Z}^{d}, d \geqq r+1$,

$$
\left\langle\left(D_{\Gamma}\right)_{r}^{d}\right\rangle_{p} \geqq\left[-\bar{c}_{r}^{r+1}(p) \operatorname{Bnd}(\Gamma)\right]
$$

with $\bar{c}_{r}^{r+1}(p)<\infty$ whenever

$$
p>1-\frac{1}{2(r+1)} \geqq 1-\pi_{c}^{r+1} .
$$

Proof. First we note that the inequality

$$
\pi_{c}^{d} \geqq \frac{1}{2 d},
$$

which is precisely the mean field bound, is easily derived using either a Lieb-Simon inequality or a Peierls' argument.

However, if the $r$-cell density satisfies $p>1-\pi_{c}^{r+1}$ (which implies that the bond probability is less than $\pi_{c}^{r+1}$, and hence that the expected size of bond clusters is finite), then an argument exactly along the lines of Sect. 3i) shows that the $r$-cells are in the boundary law phase.

Theorem 6.7. For any hyperrectangular loop of $(r-1)$-cells on $\mathbb{Z}^{d}, d \geqq r+1$,

$$
\left\langle\left(D_{\Gamma}\right)_{r}^{d}\right\rangle_{p} \geqq\left[-\bar{c}_{r}^{d}(p) \operatorname{Bnd}(\Gamma)\right]
$$

with $\bar{c}_{r}^{d}(p)<\infty$ whenever

$$
p>\bar{p}_{r}(d)=[\log [2(r+1)]]^{1 /(2 r+1)}\left[\frac{r+1}{\bar{d}-(r+1)}\right]^{1 /(2 r+1)} .
$$

Proof. We use an argument exactly along the lines of Theorem 6.3. First, we treat the case in which $d$ is a multiple of $r+1$. We find that the effective $r$-cell probability for the $(r+1)$-dimensional system is

$$
P_{\text {eff }}=p+(1-p)\left[1-\left(1-p^{2 r+1}\right)^{[d-(r+1)] /(r+1)}\right] .
$$

Then, by Proposition 6.6, the system is in the boundary law phase whenever $P_{\text {eff }}$ $>1-\frac{1}{2(r+1)}$. This is satisfied if

$$
p>[\log [2(r+1)]]^{1 /(2 r+1)}\left[\frac{r+1}{d-(r+1)}\right]^{1 /(2 r+1)} .
$$


If $d$ is not a multiple of $(r+1)$, we replace $d$ by $\bar{d}$, the largest multiple of $r+1$ smaller than $d$.

This theorem shows that, for fixed $r$, the boundary phase dominates as $d$ tends to infinity.

\section{iii) Comments on the Phase Structure in Percolation Models}

Here we note that for a system of $r$-cells in $d>3$, it remains to be shown whether the system is always in opposite phases, in the sense that boundary law for $r$-cells at density $p$ implies and is implied by volume law for $(d-r)$-dells at density $1-p$. It has been proved that this is indeed the case for $d=2[3,4,6]$. Modulo our conjecture, this result is also true in $d=3$. The non-existence of intermediate phases is the natural generalization of Harris' theorem [6] and its converse [17].

The conjecture that dual objects are always in opposite phases at first seems to conflict with our result (Theorem 6.7) that in large dimensions, $r$-cells obey a boundary law at asymptotically low density. However, Theorem 6.7 does not imply that $r$ - and $(d-r)$-cells are simultaneously in boundary phase. The correct interpretation is that for $d \gg r,(d-r)$-cells are typically in the volume phase since their codimension is relatively small.

Of particular interest are random $\frac{d}{2}$ cells in even dimension $d$. Clearly, if no intermediate phase exists in such a self-dual system, the transition point is $p=1 / 2$. The most promising model for future study is random plaquettes in $d=4$.

\section{Relation to Interacting (Gauge) Systems}

In this section we would like to briefly point out that stochastic geometric effects like those analyzed in this paper are of utmost relevance for very familiar interacting systems. We also describe a possible surface "roughening transition" an effect which is related to the roughening transition purported to occur in the three dimensional Ising model. In either system, the actual proof of the existence of a transition is still an open problem.

\section{i) $\left\langle W_{\gamma}\right\rangle$ and the Wilson Loop}

Geometric effects play a role in various systems, and can be presented in a number of ways. The example which will be mentioned here is the $\mathbb{Z}(2)$ lattice gauge theory. Its basic variables are the edge spins, $\sigma_{b}$, which take values in $\mathbb{Z}(2)=\{-1,+1\}$. The action is the sum over plaquettes:

$$
\mathscr{A}=\sum_{Q} \prod_{b \in \partial Q} \sigma_{b}
$$

The action, and the corresponding Gibbs state, are invariant under the gauge transformation $\sigma_{\{i, j\}} \rightarrow(-1)^{k_{\imath}+k_{j}} \sigma_{\{i, j\}}$. Due to this symmetry, the single-spin expectation values, $E\left(\sigma_{b}\right)$, vanish. The gauge invariant quantities are generated by the 
variables

$$
\sigma_{\gamma}=\prod_{b \in \gamma} \sigma_{b} .
$$

which are associated with loops of lattice edges.

Of central interest in this model is the expected value of the "Wilson loop" variable $\sigma_{\gamma}$, for large loops $\gamma$. The area law for this expectation corresponds to a phase of the gauge field in which static charges are "confined" - being bound by a linearly growing potential, whereas in the perimeter regime their dissociation energy is finite.

The analogy between the asymptotic behavior of the Wilson loop variable to $\left\langle W_{\gamma}\right\rangle$ is not accidental! It turns out that $E\left(\sigma_{\gamma}\right)$ is directly related to $\left\langle W_{\gamma}\right\rangle$, albeit in a system of interacting plaquettes.

The main part of the interaction is a constraint which projects the Bernoulli measure on the ensemble of those configurations whose occupied plaquettes form closed surfaces, i.e. configurations which have no boundary [in the sense of Eq. (2.2)]. The additional interaction, which is needed for an identity between $E\left(\sigma_{\gamma}\right)$ and $\left\langle W_{\gamma}\right\rangle$, is far less singular, and may even be entirely eliminated by a method which involves duplication. The result is an exact relation, for whose details, and some applications, the reader is referred to [8].

\section{ii) A Roughening Transition}

A geometric representation of a $\mathbb{Z}(2)$ system occurs already at the level of the Ising model. In fact, one of the model's properties which has not yet been fully elucidated is the "roughning transition," which in $d=3$ dimensions is purported to occur strictly below the critical temperature $T_{c}$. The transition is manifested in the destabilization of the "interface" between the two phases. A stable phase coexistence is described by a Gibbs state which is not invariant under translations. At low temperatures (and zero magnetic field) such a state can be induced by Dobrushin's anti-symmetric $(+/-)$ boundary conditions [18]. At a temperature $T_{R}$ the interface destabilizes, and the $(+/-)$ state becomes translation-invariant. We shall now describe a possible phenomenon in the random plaquette system, which offers an analog of the above transition.

Let $\Lambda_{n}=[-n, n]^{3} \subset \mathbb{Z}^{3}$ be a three-dimensional cube, whose boundary we partition into

$$
\partial \Lambda_{n}^{+}=\left\{(x, y, z) \in \partial \Lambda_{n} \mid z \geqq 0\right\},
$$

and a similarly defined $\partial \Lambda_{n}^{-}$. The intersection

$$
\gamma_{n}=\partial \Lambda_{n}^{+} \cap \partial \Lambda_{n}^{-}
$$

is a loop lying in the $\{z=0\}$ plane.

We shall discuss random configurations of plaquettes in $\Lambda_{n}$, conditional on the event $W_{n}$ - which is said to occur if there is a subset of "occupied" plaquettes, in $\Lambda_{n}$, which forms a surface $S$ with $\partial S=\gamma_{n}$.

As in the bulk of this paper, we associate with each configuration of plaquettes of $\Lambda_{n}$ a complementary configuration of bonds of the dual set $\Lambda_{n}^{*}$. For each site $x \in \Lambda_{n}^{*}$, let $T_{x, n}^{+}$denote the event that there is a path of occupied bonds which connects $x$ with the $\partial \Lambda_{n}^{+}$half of the boundary. $T_{x, n}^{-}$is defined analogously, with $\partial \Lambda_{n}^{-}$. 
Clearly, $W_{n}$ is not compatible with the occurrence of both $T_{x, n}^{-}$and $T_{x, n}^{+}$. We now propose to consider the quantities

$$
\tau_{p, n}(x)=\mu_{p}\left(T_{x, n}^{+} \mid W_{n}\right)-\mu_{p}\left(T_{x, n}^{-} \mid W_{n}\right),
$$

and

$$
\tau_{p}(x)=\lim _{n \rightarrow \infty} \tau_{p, n}(x),
$$

where the probabilities refer to the Bernoulli ensemble of plaquettes of density $p$, conditional on the event $W_{n}$.

For $p>1-p_{c}$ :

$$
\tau_{p}(x)=0 \quad \text { for every } x,
$$

for the simple reason that the dual bonds do not percolate. An application of the FKG inequality shows that each of the two terms in (7.6) vanishes in the limit $n \rightarrow \infty$.

However, for $p<1-p_{c}$ we do not expect the two terms in (7.6) to vanish individually. Here the vanishing of $\tau_{p}(\cdot)$ would instead be an indication of a nontrivial cancellation. Such a cancellation should occur precisely when the separating surface, the existence of which is imposed by the conditioning on $W_{n}$, fluctuates (in the limit $n \rightarrow \infty$ ) away from every fixed point $x$. The analogy with Dobrushin's result for the Ising model suggests that at sufficiently small $p$ (and $d>2$ ) the surface remains tight - developing only local fluctuations, and

$$
\tau_{p}(x) \neq 0 \text {. }
$$

We introduce therefore the roughening density, $p_{R}$, as:

$$
p_{R}=\sup \left\{p \in[0,1] \mid \tau_{p}(\cdot) \neq 0\right\} .
$$

A roughening transition is said to occur if $p_{R}$ obeys the strict inequalities

$$
0<p_{R}<1-p_{c}
$$

The sense in which the quantities $\tau(x)$ and $p_{R}$ are analogous to the Ising models $\left\langle\sigma_{x}\right\rangle_{ \pm}$and $T_{R}$ should be clear from the geometric representation of the latter in [8]. A proof of (7.10), which is of an independent interest, may shed some light on the general phenomenon of surface roughening.

\section{References}

1. Polyakov, A.M.: Quantum geometry of bosonic strings. Phys. Lett. 103B, 207 (1981)

2. Durhuus, B.: Quantum theory of strings. In : Lecture Notes, Nordita-82/36, and references therein

3. Kesten, H.: On the time constant and path length of first-passage percolation. Adv. Appl. Prob. 12, $848(1980)$

4. Kesten, H.: The critical probability of bond percolation on the square lattice equals $\frac{1}{2}$. Commun. Math. Phys. 74, 41 (1980)

5. Russo, L.: On the critical percolation probabilities. Z. Wahrscheinlichkeitstheorie Verw. Geb. 56, 229 (1981)

6. Harris, T.E.: A lower bound for the critical probability in certain percolation processes. P. Camb. Philos. Soc. 56, 13 (1960) 
7. Wierman, J.C.: Bond percolation on honeycomb and triangular lattices. Adv. Appl. Prob. 13, 293 (1981)

8. Aizeman, M.: Surface phenomena in Ising systems and $\mathbb{Z}(2)$ gauge models (Geometric analysis, Part III) (in preparation)

9. Fortuin, C., Kastelyn, P., Ginibre, J.: Correlation inequalities on some partially ordered sets. Commun. Math. Phys. 22, 89 (1971)

10. Kesten, H.: Analyticity properties and power law estimates of functions in percolation theory. J. Stat. Phys. 25, 717 (1981)

11. Krinsky, S., Emery, V.: Upper bound on correlation functions of Ising ferromagnet. Phys. Lett. 50A, 235 (1974)

12. Simon, B.: Correlation inequalities and the decay of correlations in ferromagnets. Commun. Math. Phys. 77, 111 (1980)

13. Lieb, E.: A refinement of Simon's correlation inequality. Commun. Math. Phys. 77, 127 (1980)

14. Aizenman, M., Newman, C.: Tree diagram bounds and the critical behavior in percolation models (in preparation)

15. Higuchi, Y.: Coexistence of infinite (*) clusters. Z. Wahrscheinlichkeitstheorie Verw. Geb. 61, 75 (1982)

16. Kesten, H.: Percolation theory for mathematicians. Boston, Basel, Stuttgart: Birkhäuser 1982

17. Russo, L.: A note on percolation. Z. Wahrscheinlichkeitstheorie Verw. Geb. 43, 39 (1978)

18. Dobrushin, R.L.: Gibbs states describing coexistence of phases for a three-dimensional Ising model. Theor. Prob. Appl. 17, 582 (1972)

Communicated by A. Jaffe

Received June 16, 1983 
